\title{
Title: Defining the microenvironment landscape of bladder cancer using highly multiplexed spatial genomic and proteomic analysis
}

Authors: Jason W Reeves ${ }^{1}$, Zhaojie Zhang ${ }^{2}$, Zachary K Norgaard ${ }^{1}$, Denise M Zhou ${ }^{1}$, JingJing Gong ${ }^{1}$, Yan Liang ${ }^{1}$, Subhasree Das $^{2}$, Sarah E Warren ${ }^{1}$, Manav Korpal ${ }^{2}$, Margaret L Hoang ${ }^{1}$, Joseph M Beechem ${ }^{1}$, Pavan Kumar ${ }^{2}$, Victoria Rimkunas ${ }^{2}$

\author{
Affiliations: \\ ${ }^{1}$ NanoString Technologies, Inc., Seattle, Washington, USA \\ ${ }^{2} \mathrm{H} 3$ Biomedicine, Cambridge, Massachusetts, USA
}

One Sentence Summary: A new method for capturing tumor-immune signaling in FFPE tissues explores how the PPARG signaling axis is associated with immune cell exclusion in bladder cancer.

\begin{abstract}
:
Muscle-invasive bladder cancer (MIBC) is an aggressive disease with limited therapeutic options. PD-1 pathway targeting immunotherapies have been approved to treat advanced bladder cancer, but most patients exhibit primary resistance, suggesting that immune evasion mechanisms exist. The PPAR $\gamma$ pathway has been identified as a potential therapeutic target in MIBC that is associated with reduced CD8+ T-cell infiltration and increased resistance to immunotherapies. We comprehensively profiled the tumor microenvironment (TME) in formalin-fixed, paraffin-embedded (FFPE) tissues from a cohort of PPAR $\gamma^{\text {high }}(\mathrm{n}=13)$ and PPRAR $\gamma^{\text {low }}(\mathrm{n}=12)$ MIBC, integrating bulk gene expression, targeted mutation sequencing, immunohistochemistry and multiplex spatial profiling of RNA and protein expression on the GeoMx ${ }^{\text {TM }}$ Digital Spatial Profiling (DSP) platform. Molecular subtyping was consistent between traditional methods and GeoMx profiling, and, in this cohort, we observed little evidence of spatial heterogeneity in tumor subtyping. The previously characterized T-cell exclusion phenotype of PPAR $\gamma^{\text {high }}$ MIBC was recapitulated on the GeoMx platform and was further extended to show that this is a general phenomenon across immune cell types, supporting potential combination of PPAR $\gamma$ inhibition with ICIs. Furthermore, we found that while immune cells were excluded from PPAR $\gamma^{\text {high }}$ tumors, the stromal compartment from these tumors was not significantly different than those PPAR $\gamma^{\text {low }}$ tumors. By preserving spatial relationships during the GeoMx analysis, we also identify a novel association between lower immune cell expression in the tumors and higher expression of $\beta$-catenin in the stroma, and differential expression of other WNT pathway members associated with PPAR $\gamma$ activity.
\end{abstract}




\section{Introduction}

Muscle invasive bladder cancers (MIBCs) are biologically heterogenous and have widely variable clinical outcomes and responses to conventional chemotherapy $(1-4)$ and immune checkpoint inhibitors(5). Although responses can be durable, only 20-30\% of patients with MIBC will respond to immune checkpoint inhibitors (ICIs), arguing for the identification of novel drug targets and need for a deeper understanding of predictive biomarkers to enrich for responses to current standard of care therapies $(6,7)$. Multiple studies have identified distinct RNA expression subtypes within MIBC termed "luminal" and "basal-like"(8), each of which has unique gene expression patterns which have important implications for disease management with conventional chemotherapy(9-11) and immunotherapies(12). Response to immune checkpoint inhibitors in urothelial bladder cancer has been associated with several potential biomarkers including tumor mutation burden, tumor molecular subtype and PD-L1 expression on CD8+ tumor infiltrating lymphocytes (TILs) and other immune cells(7, 13-15).

Patients with the luminal I subtype rarely respond to $\operatorname{ICIs}(14,15)$, suggesting the existence of one or more immune escape mechanisms. We previously reported that the PPAR $\gamma /$ RxRa pathway constitutes a tumor-intrinsic mechanism underlying immune evasion in $\operatorname{MIBC}(6,16)$. In tumors with high PPAR $\gamma$ pathway activity, CD8+ T-cell infiltration appears to be impaired through chemokine suppression, though additional mechanisms may also impact Tcell infiltration. These findings suggest that pharmacological inhibition of PPAR $\gamma$ may induce sensitivity to immunotherapies. To further our understanding of tumor-immune escape mechanisms in MIBC, analysis of both the tumor and the surrounding microenvironment is necessary. Comprehensive interrogation of the interaction between these two compartments has proved challenging thus far because most of the foundational work in MIBC has relied on commonly available assays such as single-plex immunohistochemistry (IHC) assays or bulk RNA expression and mutation profiling. With limited availability of tumor tissues during clinical trials, highly multiplexed and quantitative platforms compatible with small formalin-fixed paraffin-embedded (FFPE) tissues are necessary to deliver a comprehensive understanding of the tumor-immune landscape.

Historically, protein assessments of the TME have been limited to PDL1 and CD8 single plex IHC staining, which is primarily driven by the observation that in urothelial carcinoma and other cancers, outcomes related to ICI treatment appear particularly favorable in patients with high PD-L1 expression(7, 13, 14, 17, 18). But these assays for PD-L1 have been developed for individual therapies, using varying antibody clones, staining protocols, scoring algorithms and cut-offs, which makes standardization across multiple studies difficult $(19,20)$. In addition, conventional IHC has significant limitations due to relatively low sensitivity for poorly antigenic or expressed targets, difficulty in colocalizing stains, and the subjective and semi-quantitative nature of pathologists' interpretations. While significant progress has been made in multiplex IHC technologies, significant challenges remain for the field. Immunofluorescence (IF) platforms such as Ultiview, Multiomyx and Opal can provide quantitative analysis of multiple stains from a single slide, but the analysis is quite complex, time consuming and costly, and except for Multiomyx, most multiplex IHC technologies are limited to 4-7 analytes per slide, which is insufficient for fully characterizing the TME.

Given the limitations of IHC, gene expression profiling is an attractive alternative allowing simultaneous profiling of hundreds to thousands of transcripts from as little as $50 \mathrm{ng}$ of 
RNA. In high quality samples, this can be extracted from a single FFPE slide, although the RNA yield varies drastically by tissue sample size, tumor type, and age of the slide. RNAseq and NanoString ${ }^{\circledR}$ nCounter ${ }^{\mathrm{TM}}$ direct hybridization are two bulk gene expression methods that have routinely been used for studying the TME, resulting in identification and validation of gene signatures like the tumor inflammation signature (TIS) $(21,22)$ showing correlation with response to ICIs in other cancers $(19,23)$. The breadth and scale of gene expression analysis has been foundational in oncology, but these methods lack the information conferred by spatial resolution of the signal. Previous studies in colorectal cancer and melanoma have highlighted the critical role that the spatial localization of the immune compartment plays in patient outcome(2325), while the role of the spatial and temporal distribution of CD8 TILs and PD1 expression has been suggested to be predictive of response to ICIs $(17,18)$. Spatial resolution of RNA biomarkers can be retained with technologies such as RNAscope (in situ hybridization, ACD) but similar to multiplex IHC, only 1-3 analytes per FFPE section can be measured. A higher resolution and multiplexing can be afforded by a number of methods in fresh frozen tissues (2628 ), but these require specialized workflows not frequently compatible with clinical trials nor are such approaches applicable to archived FFPE tissues.

In this report, we describe the application of multiple bulk analysis and spatial multiplexed technologies to characterize the TME in a cohort of MIBC FFPE tumor samples, using tissue stored in FFPE blocks previously used to characterize the CD8 immune-exclusion phenotype associated with PPAR $\gamma$ activity (6). For bulk profiling, we applied both RNA sequencing and NanoString PanCancer IO 360 gene expression readouts, as well as a targeted sequencing panel for common cancer-associated mutations. To resolve spatial interactions, we processed samples using the NanoString GeoMx platform using both multiplexed RNA in situ hybridization (ISH) and a multiplexed antibody cocktail, while using traditional single-plex IHC or ISH for validation purposes. This platform uses UV photocleavable oligonucleotide tags conjugated antibodies or RNA-ISH probes to interrogate targets expression in a tissue section. The oligonucleotides are released from specific regions of interest (ROIs) via exposure to UV light, in paths that are controlled by a digital micromirror array $(29,30)$. The goals of this study were to interrogate the molecular composition of the TME of MIBC across complementary technologies, and to characterize the ability of different platforms to profile clinical samples where tissue is limited. We also explore the role of tumor-stromal interactions within this cohort to expand our understanding of potential ICI resistance mechanisms and opportunities for therapeutic combinations in advanced bladder cancer.

\section{Results}

\section{Bulk and spatial approaches for understanding tumor-stromal interaction in MIBC}

To investigate the interplay between tumor molecular subtype, PPAR $\gamma$ signaling, and the composition of the TME, we profiled a previously collected cohort of 25 samples from advanced stage MIBC for which PPAR $\gamma$ pathway activity and CD8 staining had been previously characterized (Table 1, Supplementary Table 1) (6). These samples had a higher proportion of male patients, a balance of samples with or without lymph node involvement, and with most samples being late stage, though 5 of the 13 PPAR $\gamma^{\text {high }}$ tumors were stage I-II. PPAR $\gamma^{\text {high }}$ samples had higher tumor cellularity on average whether estimated on either the original H\&E ( $p$ $=0.04$, Welch's t-test, Supplementary Table 1$)$ or IF channels from RNA $(p<0.008$, Welch's ttest, Supplementary Table 1). The workflow used to characterize this cohort included a multifaceted approach of combining traditional bulk sequencing and expression assays with 
spatial profiling by IHC/ISH assays and multiplexed protein and RNA quantification on the NanoString GeoMx platform (Figure 1A).

For each sample, the GeoMx workflow involved selection of regions of interest (ROIs), which can be quantitated using downstream sequencing or nCounter readouts. In this study, we selected 5-6 ROIs per patient by focusing on areas of the slide with both tumor-rich and stomal areas within the same ROI using Pan-CK and CD3 IF staining to assess tumor and stromal content, avoiding regions of necrosis or tissue detachment (Supplementary Figure 1). Examples of multiplexed IF images used to guide UV illumination are shown based on PPAR $\gamma$ status in (Figure 1B). Custom UV illumination masks were designed to create areas of illumination (AOIs) that result in photocleavable tags being released specifically in either tumor (Pan-CK+) or stromal (Pan-CK-) compartments, a process called auto-segmentation $(29,30)$ (Figure 1A). 23 samples of the cohort were profiled using multiplexed antibodies, collecting tags from 273 AOIs. Two samples failed due to tissue detachment during processing. 19 samples were profiled with multiplexed RNA-ISH collecting tags from 222 AOIs, after 3 samples failed due to tissue detachment. The tag oligonucleotides were quantified using either the nCounter platform, for antibody tags, or with next generation sequencing (NGS), for RNA-ISH tags. GeoMx measured expression of 40 proteins or 157 RNA transcripts simultaneously, using a single five-micron FFPE slide for each type of analyte. Of the AOIs profiled by RNA-ISH, 13 were flagged as having high background and removed from analysis (Supplementary Table 2, Supplementary Figure 2). 29 out of 38 protein targets stained were detected above the expression of the two $\operatorname{IgG}$ control antibodies (Supplemental Figure 1, Supplementary Data File 1). Similarly, for RNA-ISH readout the negative control probes were on average expressed at the $12^{\text {th }}$ percentile $\left( \pm 6 \%, \sigma_{x}\right)$ of all probes tested after excluding samples with high background (Supplementary Figure 2, Supplementary Data File 2).

In addition to GeoMx analysis, sections were stained for CD8 and granzyme B by singleplex IHC. IHC images of CD8 expression from the same block, though not serial section, are shown for comparison with the IF images used to guide ROI selection (Figure 1C). CD8A was confirmed to be higher in tumor AOIs that stained positive for the CD8 TILs presence ( $\mathrm{p}=$ 0.006, mixed effect model) and trended towards higher expression in stromal AOIs ( $\mathrm{p}=0.057$, mixed effect model, Figure 1D). Since CD3 was not used to create UV illumination masks but rather to help guide ROI selection, CD8 expression within tumors AOIs was attributed to CD3+ cells present in tumor areas observed by IF in samples. Similarly, PPARG expression level detected by RNA-ISH GeoMx was highest in tumor AOIs from PPARG+ tumors identified by IHC ( $p<0.05$, mixed effect model, Figure 1E). Expression of markers associated with IF staining guide followed expected patterns based on IF images, with Pan-CK expression being significantly higher in tumor AOIs than stromal AOIs $(\mathrm{p}<0.001$, mixed effect model, Figure $1 F)$. Finally, CD3 expression was more complex, with detection at equivalent levels in stromal AOIs, but significantly lower expression in tumor AOIs specifically from PPAR $\gamma^{\text {high }}$ tumors. This is consistent with the previous described exclusion phenotype(6), as CD3 IF staining was not used during auto-segmentation.

\section{Tumor molecular subtyping and intrinsic signaling of PPAR on GeoMx}

Since molecular subtype has been implicated in response to multiple therapies, it was important to understand the distribution of samples in this cohort and whether GeoMx profiling could classify the MIBC cohort into luminal or basal tumor intrinsic subtypes. To provide a baseline for analysis, we classified samples based on bulk RNA sequencing data into basal or 
luminal genomic subtypes(10). 17 samples were classified as luminal (68\%) and 8 as basal (32\%, Table 1), with membership skewed based on PPAR $\gamma$ status as previously reported from analysis of TCGA(6). CD44, a basal MIBC marker, was the only subtype marker included in both protein and RNA-ISH assays. In the protein assays, we calculated the receiver-operating characteristic (ROC) curve and found that CD44 was more predictive in tumor AOIs (AUC =0.89) than stromal AOIs (AUC $=0.66$, Figure 2A).

For RNA-ISH analysis, we first qualitatively explored the performance of probes to identify each subtype by unsupervised hierarchical clustering across the tumor AOIs. Subtype markers cluster tumor AOIs into basal and luminal tumors as classified by bulk RNA sequencing with AOIs within sample as well (Figure 2B). While the clustering was not completely resolved by subtype, the targeted panel for ISH only contained 12 subtyping markers previously described, and it is possible that profiling additional markers would provide better resolution.

To quantify the predictive power of RNA-ISH profiling to classify samples, we calculated a ROC curve for each gene. We found that markers for subtype generally performed well individually in tumor AOIs (Figure 2C), though basal markers were more consistently predictive than luminal markers. Consistent with the protein analysis, these markers were less predictive in stromal AOIs, although some keratins remained highly predictive (Figure 2D). Testing of intratumor heterogeneity among subtyping markers revealed that the average variation in expression was always higher within a given subgroup than within a sample, and that variance was equivalent between tumor and stroma AOI (Figure 2E). This suggests that intra-tumoral heterogeneity in tumor-intrinsic signaling is not likely a major factor in the AOIs sampled in our cohort.

\section{Bulk analysis masks expression in stromal compartments}

Based on the observation that subtyping genes were more predictive in the tumor AOIs, we explored the source of expression derived from bulk assays. Tumor purity, and its impact on expression profiling, has been studied in the context of the TCGA, and bladder cancers were found to have higher signal from the stroma than other many cancers (31). However, it's unclear if these bulk studies reflect subtype-specific TME structural differences, or true stromal expression patterns. Therefore, we compared our RNA-ISH results to the bulk RNA profiling by the nCounter PanCancer IO 360 assay as these were the most similar in terms of probe design and analysis workflow. We tested whether either type of AOI, Pan-CK+ tumor or Pan-CKstroma, was better correlated with bulk profiling and tested the impact of tumor cellularity on the correlation observed with either AOI type. We were able to profile 108 genes across both platforms. We found that tumor AOIs had significantly higher correlation with bulk expression within a given patient across all shared genes in the two platforms $(\mathrm{p}=4.8 \mathrm{e}-4$, Welch's t-test, Figure 3A). Patients with higher tumor cellularity as estimated from the whole-slide IF showed a slight, though insignificant, increase in correlation between bulk tumor expression and tumor AOIs ( $p=0.11$, Welch's t-test), and no appreciable difference within stromal AOIs $(p=0.26$, Welch's t-test).

Similarly, we found that, on a gene level, expression was more highly correlated with bulk expression in tumor AOIs (mean $\rho=0.56$, Spearman) than stromal AOIs (mean $\rho=0.20 ; p$ $<$ e-16, Welch's t-test, Figure 3B, Supplementary Figure 3). Higher average gene expression in tumor AOIs was also moderately associated with higher correlation with bulk expression $(\rho=$ 0.55, Supplemental Figure 3). When assessing genes from different pathways or gene sets, genes 
that were part of tumor-intrinsic subtyping gene sets were the most highly correlated between tumor AOIs and bulk expression (Figure 3C, Supplementary Figure 3), though most gene sets followed the same pattern. Collectively, these data suggest that bulk profiling of bladder cancer more closely reflects the tumor compartment and under-represents expression from the surrounding stroma.

\section{Spatial organization of TILs in MIBC associates with PPARy expression}

To further explore the biologic basis of immune exclusion in our cohort, we performed Gene Set Enrichment Analysis (GSEA) on the RNA-sequencing data with cancer hallmark signatures (32). Immune related hallmarks, such as inflammatory response, IL6/JAK/STAT3 signaling, IL3/STAT5 signaling and TNFA signaling are enriched in the PPAR $\gamma^{\text {low }}$ group (Figure 4A), while PPAR $\gamma$ signaling-related signature such as Oxidative Phosphorylation and Fatty Acid Metabolism were anticorrelated with this group (Supplementary Data File 3). In addition, results from IO 360 profiling of the TIS $(17,20)$ found that TIS was anticorrelated with PPARG expression ( $\rho=-0.66$, Spearman, Figure 4B). TIS expression was positively correlated with CD8+ TIL infiltration $(\rho=0.72$, Spearman) and PD-L1 expression $(\rho=0.91$, Spearman $)$, while PPARG RNA expression was inversely associated with both $(\rho=-0.43,-0.73$ respectively, Spearman). In addition to TIS, nearly all immune-related signatures calculated from IO 360 were lower in PPAR $\gamma^{\text {high }}$ tumors (Supplementary Data File 3), suggesting this exclusion extends beyond the T-cell infiltration previously characterized (Figure 4C).

To identify immune exclusion and differentiate this from the tissue being devoid of immune cells (i.e. an immune desert), we compared the expression of protein or RNA markers between tumor and stromal AOIs, while controlling for inter-patient variability in expression in either PPAR $\gamma^{\text {high }}$ or PPAR $\gamma^{\text {low }}$ tumors. We found that PPAR $\gamma^{\text {high }}$ tumors, but not PPAR $\gamma^{\text {low }}$ tumors, exhibited significant differential expression of immune cell markers. PPAR $\gamma^{\text {high }}$ samples had higher expression in the stromal AOIs after accounting for multiple testing (FDR $<0.05$, Figure 4D, Supplementary Table 3). Markers enriched in the stromal compartment included numerous cell type markers, including lineage specific markers such as CD8A, CD56/NCAM1, ICAM1, CD14, CD68, and CSF1R. Furthermore, many immune signaling markers including cytokines and chemokines measured as part of the RNA-ISH DSP were found to be restricted to the stroma in PPAR $\gamma^{\text {high }}$ tumors, as anticipated since immune trafficking is impaired in these tumors. In contrast, while nominal differential expression was observed between tumor and stroma AOIs for PPAR $\gamma^{\text {low }}$ tumors, no immune cell markers reached significance after adjusting for multiple testing (FDR <0.05).

While the immune exclusion phenotype has been previously demonstrated, it was unclear whether the composition of immune cells in the stroma was different between the two subtypes of the disease. We examined this by comparing the expression of markers in the stromal compartments between PPAR $\gamma^{\text {high }}$ and PPAR $\gamma^{\text {low }}$ tumors. Under this analysis, few markers reached significance after adjusting for multiple test correction in either the protein or RNA dataset, even while using a less stringent false discovery rate (Figure 4E, FDR < 0.1). We also ran unsupervised hierarchical clustering of antibodies related to immune cell types or signaling from stromal AOIs and compared the results to similar analysis of tumor AOIs (Figure 4F). Under this analysis we found that PPAR $\gamma$ groups cluster based on tumor AOIs expression, while stromal AOIs did not have a similar clustering structure. Moreover, under traditional IHC analysis we did not see significant differences in GZMB expression based on PPAR $\gamma$ expression (Supplemental Figure 4), suggesting no differential impact on the cytolytic potential in cells 
neighboring the tumor. Taken together these results suggest that PPAR $\gamma$ expression does not drive changes in composition of the immune cells outside the tumor, though its expression is related to their exclusion.

\section{High tumor mutation burden found in PPAR $\gamma^{\text {high }}$ patient sample with high TIL presence}

Despite the immune-exclusion mechanisms associated with PPAR $\gamma$ expression, we identified one patient sample, 10182, expressing high PPAR $\gamma$ while also having high TIS and increased TIL presence by IF or IHC staining (Figure 5A-B). This was reproduced in GeoMx analysis, showing that CD3 protein expression was similar between tumor and stromal AOIs, and observed at levels similar to those of PPAR $\gamma^{\text {low }}$ tumors (Supplemental Figure 5). One possible explanation for an inflamed TME is a high tumor mutation burden which others have shown may be an orthogonal metric for immune activation from general tumor infiltration and inflammatory signaling (23). As this would not be directly captured in any of the other assays run, we profiled samples on the TSO-500 targeted sequencing panel, comprising 500 commonly altered genes in cancer. From this assay, tumor mutational burden (TMB) was estimated based on the rate of nonsynonymous mutations observed in the panel targets.

While we found that TMB was not significantly different based on PPAR $\gamma$ expression, we found that sample 10182 had the highest TMB within PPAR $\gamma^{\text {high }}$ tumors (Figure 5B-C). We also found that in addition to a high mutational load, there were also specific mutations in genes related to immune signaling pathways including PDCD1, STAT3, and SMAD4 (Figure 5D). Of particular note, the SMAD4 mutation, T338I, was found within the MH2 functional domain and was predicted to be oncogenic (0.92, Cscape score) (33). Signaling within the TGF $\beta$ pathway, which is may be impacted by this mutation, has been reported to impact TIL presence and response to ICIs (34). Together, both TMB and specific mutations may represent compensatory mechanisms allowing TIL invasion despite PPAR $\gamma$ signaling in this patient.

\section{Stromal $\beta$-catenin signaling inversely associated with TILs identified by spatial genomics approaches}

As TIL exclusion was related to tumor-intrinsic PPAR $\gamma$, we wanted to further explore interactions between the tumor and stromal compartment that would be missed by bulk profiling or subgroup specific analysis. Because we profiled samples with matched tumor-stroma pairs of AOIs ( $n=95$, paired AOIs after QC), we designed a framework to test whether the tumor-stroma relationship between any sets of genes on the RNA-ISH panel was not observed in their native compartment and might be the result of paracrine or juxtacrine signaling (Figure 6A). Specifically, we calculated whether the tumor expression of a gene, $T$, was correlated with stromal expression another gene, $S(|\rho(T, S)|>0.5$, Spearman), but not when comparing expression in the same compartment $(|\rho(T, T ; S, S)|<0.2$, Spearman). This allows us to detect potential interactions that subgroup-based analyses might miss. For example, we found that CTNNB1 ( $\beta$-catenin) expression in stromal AOIs was anticorrelated with PTPRC (CD45) expression in the tumor AOIs $(\rho=-0.56)$, but not in any other combination of AOI comparisons (Figure 6B).

Across all genes on the panel, we found that 119 pairs of genes exhibited this type of tumor-stroma specific correlation. As these interactions could be driven by patient-specific expression, we followed this analysis with an additional regression accounting for the patient from which an ROI was collected. Of the identified gene interactions, 65 were significant after accounting for patient-specific expression and multiple testing (FDR $<0.05$, linear mixed effect 
model, Supplementary Table 3). The most frequently identified gene was CTNNB1 expression in stromal AOIs. CTNNB1 was anticorrelated with tumor expression of 28 other genes, most of which were noted to be immune cell markers or signaling molecules (Figure 6C). The next most common interaction partner identified was stromal expression of EPCAM, which had 12 significant interactions. While the antibody panel contained $\beta$-catenin and several of the immune cell targets identified in this analysis, we were unable to validate this finding in the protein GeoMx data (Supplementary Table 3). We attribute this to the fact that $\beta$-catenin localization, reflective of $\beta$-catenin activity, is not captured during GeoMx analysis, confounding our interpretation of the antibody data. Furthermore, analysis of bulk expression showed that tumor expression of CTNNB1 was correlated with bulk profiling $(\rho=0.69)$, suggesting accurate measurement of the target since CTNNB1 was observed to have higher expression in the stroma than in tumor compartment, though this was not significant after adjusting for multiple testing and patient-specific expression (FDR $=0.15$, mixed effect model, Figure 6D).

To better understand the potential role of the $\beta$-catenin signaling axis in these tumors, we returned to the expression of other genes on the RNA-ISH panel which were not specifically identified by the previous analysis. We noted that several genes in the WNT/ $\beta$-Catenin signaling pathway were identified as significantly enriched in the stroma of PPAR $\gamma^{\text {high }}$ tumors (Figures 3D). One of the members of this pathway, GSK3B, had similar patterns of expression across sample types as $\beta$-Catenin itself, and was specifically higher in PPAR $\gamma^{\text {high }}$ stroma (FDR $=6.4 \mathrm{e}-3$, mixed effect model, Figure 6D). Of the 6 frizzled-family receptors on the RNA-ISH panel, all except FZD3 had higher median expression within the stroma of PPAR $\gamma^{\text {high }}$ tumors (Figure 6F). Additionally, other WNT/ $\beta$-Catenin targets, such as CCND2/3, CREBBP, and APC were all similarly highest in stroma of PPAR $\gamma^{\text {high }}$ tumors, though expression was observed in PPAR $\gamma^{\text {low }}$ stroma as well. The differential expression of these genes appears to be driven by very low expression within the tumor compartment.

\section{Discussion}

The study we report here follows the analysis of a cohort of advanced stage MIBC. In this setting, understanding the molecular underpinnings of a sample is critical to better predicting how they will respond to therapy(10), especially $\operatorname{ICIs}(12,13)$. To better characterize the spatial arrangement of key molecular characteristics such as tumor-intrinsic subtype, TIL exclusion and composition, and tumor-stroma interaction we leveraged multiple traditional bulk and spatial platforms in conjunction highly multiplexed profiling by GeoMx within FFPE tissues. This allowed us to deeply explore signaling within and around the tumor, and also how it varied across the sample.

Molecular subtyping has traditionally been performed using RNA expression profiling of bulk tissues from MIBC samples $(8,9,16)$. When compared to matched bulk profiling by RNA sequencing, we were able to robustly measure MIBC subtype markers with high predictive power in either the RNA or protein GeoMx assays. This demonstrates that from a single slide, it may be possible to capture and classify samples into subtype. Contamination of surrounding tissue may impact robustness of molecular classification. In our GeoMx experiment, this is avoided directly using auto-segmentation, allowing the investigation of compartment specific expression without having to manipulate the tissue or rely on pathological identification of tumor boundaries. Indeed, we noticed that stromal AOIs had significantly worse predictive performance, and application of auto-segmentation or strategic ROI placement is necessary for robust classification. 
Furthermore, we observed little evidence of intra-tumor heterogeneity in molecular subtype markers. This is in contrast to recently published work suggesting that some tumors exhibit a high degree of intra-tumoral heterogeneity $(35,36)$. However, we note this difference may be due to definitions of subtype applied, the profiling strategy of this and other studies, and the small numbers of samples tested in each study. As our study was limited to 25 samples, we focused on detecting the differences between luminal and basal type tumors, as we would not be powered for further stratifications of the disease. We did note enrichment of TP53 mutations in specific luminal samples, however, without additional markers on the RNA-ISH panel we were unable to more deeply stratify samples.

In addition to subtype, the TME of MIBC is a critical component to patient outcome. We recapitulated the previously reported T-cell exclusion phenotype which was described in tumors expressing high levels of PPAR $\gamma(\sigma)$. Specific exclusion of immune cell markers in the tumor compartment of PPAR $\gamma^{\text {high }}$ samples was observed across nearly all immune markers present on the panels in both the protein and ISH GeoMx studies. By directly measuring expression from stromal compartments, we were also able to assess whether the stromal immune composition of the PPAR $\gamma^{\text {high }}$ tumors were qualitatively different from PPAR $\gamma^{\text {low }}$ tumors. We found little evidence of differential expression between tumor based on PPAR $\gamma$ status, and genes that we did find to be differentially expressed were not generally immune-cell related. This supports the potential to improve ICI response by targeting the PPAR $\gamma$ pathway in these tumors, as this may remove the barrier to immune cell invasion in these tumors.

By spatially resolving the source of expression in a tissue, we were able to directly determine whether bulk profiling of tissues reflects a specific compartment within the sample, and identify novel interaction networks within and between tissue compartments. The bulk expression profiles were most similar to the tumor compartment, which may be due to the hyperproliferative nature of tumor cells. However, interrogation of other tissue types will be necessary to see if this results in a phenomenon that is broadly observed or specific to MIBC. In silico analysis of the TCGA suggest some tumor types may have more stromal signaling(31). Our findings suggest that even in indications with a high stromal content measured by bulk profiling, these studies may underestimate the role that the TME plays in tumor development by underrepresenting expression from the stroma itself. The observation in TCGA analysis that bladder cancers had lower tumor purity than many tumor types likely reflect the highly infiltrated phenotype of PPAR $\gamma^{\text {low }}$ tumors. In contrast, by directly measuring the stroma, we can begin to understand interactions between the compartments within that tissue. It is currently unknown if stromal signaling within MIBC may be related to tumor evolution and how to best target these tumors with therapeutics. The correlation analysis we employed suggests stromal expression of $\beta$-catenin may also be related to exclusion of immune cells in addition to tumor intrinsic PPAR $\gamma$ expression. While the functional role of $\beta$-catenin expression the stroma is unclear, these findings are reminiscent of work demonstrating the role of this pathway in immune cell exclusion in melanoma (37).

Beyond the key findings within MIBC, this study demonstrates the technical feasibility of using two sections of FFPE tissue per sample to obtain a highly nuanced view into the tumorimmune landscape. While this study was performed on archival tissue blocks, it provides a proof of principle that this technology can be applied to FFPE samples with limited material. Other technologies which address both location and multiplexing of analytes have distinct limitations in their ability to impact translational applications to clinically relevant samples such as 
degradation of signal during multiplexing, technical variability, and cumbersome downstream data analysis. Moreover, multiplexing of RNA probes in FFPE tissues has been a hurdle which few technologies have overcome. Technologies which offer the potential of higher multiplexing are limited to fresh-frozen tissues (25-27), and previously only low plex ISH approaches were compatible with the FFPE tissue which are most commonly available from the clinic. GeoMx RNA-ISH profiling offers a solution for high plex exploratory research on FFPE tissue that could be translated to low plex clinical assays on a diagnostic platform.

As GeoMx represents a complimentary approach for single-plex or multiplexed IHC and ISH, there are some differences between this platform and conventional profiling platforms that should be taken into consideration during experimental design. First, GeoMx profiling measures target abundance in an IF defined areas, which enables profiling of cell populations rather than individual cells. Second, as target quantification is mediated by direct probe hybridization, the platform is not capable of measuring somatic mutation, and assays capturing oncogenic mutations and TMB remain a complementary approach. This was demonstrated in our study, as one PPAR $\gamma^{\text {high }}$ sample, 10182, had higher TMB and a specific SMAD4 mutation (T338I) that may explain the immune infiltration observed in this tumor, given the known impact of TGF $\beta$ signaling in immune infiltration and ICI response(34). Finally, GeoMx profiling does not require cycling to achieve high plex, where stripping and re-probing with new antibodies tends to degrade signal over time. Rather the quantification of all targets is performed simultaneously and maintains their relative abundance.

While this study highlights several key advantages of performing GeoMx on clinically relevant samples, we note that there are several limitations to our study. As we only tested 157 genes in the RNA-ISH and 40 antibodies, there are a significant number of pathways or targets of interest that were not measured. Also, while this manuscript presents some preliminary strategies to capture the cell-type diversity and interactions between tumor and stromal compartments, statistical methodologies will need to be adapted for the unique characteristics of the data being generated on this platform. For example, methodologies aimed at cell-type deconvolution, including CIBERSORT $(38,39)$, TIMeR (40), and MuSIC (41), have been shown to capture the cellular composition of bulk-profiled samples. These algorithms may need to be adapted for spatial profiling by incorporating AOI-specific information such as AOI size or number of cells. Other spatially resolved platforms have started to propose modification to differential expression analysis to leverage the spatial data captured(42). Due to the sparse collection strategy employed such methods may need to include additional caveats to be applied to GeoMx profiling data.

With rapid advances in the ability to profile tissues in a spatially resolved manner, we have found that GeoMx provides a robust, targeted platform for profiling tissues that is readily geared towards exploratory studies. Incorporating it with multiple platforms in this study allowed us to extend beyond previous foundational studies, and deeply profile the tumor-stroma interaction in MIBC. Such profiling strategies have a clear benefit for translational researchers allowing spatial profiling while maintaining high-plex quantification of FFPE samples.

\section{Materials and Methods}

\section{Tissue Procurement}

25 formalin-fixed, paraffin-embedded (FFPE) samples from bladder cancer patients were purchased from Proteogenex and banked at Eisai (Andover, MA). Informed consent was 
obtained from all patients by the Russian Oncological Research Center n.a. N.N. Blokhin Rams Ethics Committee.

\section{Bulk RNA profiling by IO 360 and RNA sequencing}

For each sample, 5 of slides were processed using the Qiagen RNeasy FFPE Kit to extract total RNA from $10 \mu \mathrm{M}$ FFPE tissue sections. RNA was profiled using the NanoString nCounter IO 360 panel, using 100 ng of RNA per sample profiled using the nCounter Max Profiler. Expression of all probes was normalized based on the housekeeping probes included in the panel such that the geometric mean of expression of the housekeeping genes for a sample was centered at a constant value of 7 in $\log _{2}$ expression space for all samples. $100 \mathrm{ng}$ RNA was also profiled using TruSeq RNA access Library Prep Kit on an Illumina Nextseq to a depth of 200 million reads with a minimum of 10 million reads. RNA-sequencing reads were mapped using the STAR aligner (43) and quantified using Kallisto (44).

\section{Immunohistochemistry and In Situ hybridization}

Immunohistochemistry Primary antibodies include granzyme B clone 11F1 (Leica Biosystems), PPAR $\gamma$ clone C26H12 (Cell Signaling Technology), CD8 clone SP57 (Ventana Medical Systems) and PD-L1 clone 22C3 (Agilent Technologies). The CD8 assay was performed on the Ventana automated immunostainer BenchMark ULTRA. The PDL1 assay was performed using EnVision FLEX HRP visualization system on DAKO Autostainer Link 48 platform. PDL1 staining was scored by a board-certified MD-pathologist at Cancer Genetics Inc. PPARg RNA-ISH was performed using the RNAscope technology in the service lab at Advanced Cell Diagnostics. CD8 IHC, granzyme B IHC and PPARG RNA-ISH staining was quantified using the digital pathology software Halo (Indica Labs). Scanned images, pathologist scores and interpretations were available to $\mathrm{H} 3$ Biomedicine Inc., for review and data analysis.

\section{Mutation profiling and TMB detection}

DNA was extracted from a single FFPE slide per sample and $40 \mathrm{ng}$ of DNA was used as input for sequencing on the TruSight Oncology 500 (TSO-500) Illumina targeted mutation profiling panel. Estimation of tumor mutational burden (TMB) was performed based on the number of estimated somatic mutations for either non-synonymous variants or all somatic alterations detected. Potential oncogenic impact of specific mutations was inferred using Cscape (33).

\section{GeoMx profiling of Antibody \& RNA-ISH expression}

Slides were prepared by incubating with a cocktail of either antibodies or ISH probes conjugated to photocleavage reporter tags. After incubation with reporter reagents, slides were incubated with IF-conjugated Pan-CK and CD3 probes, as well as a DNA stain. Samples were imaged on the GeoMx digital spatial profiler platform, wherein an IF microscope was used to guide live image selection of regions of interest placement. AOIs were created from ROI images using PanCK IF staining, creating PanCK+ and PanCK- segments. UV light was shown onto the tissue based on the segments defined by PanCK staining using a digital micromirror array, and tag oligos were collected for downstream quantification. Antibody conjugated tags were profiled using the nCounter Flex system, while the RNA-ISH probes were quantified using an Illumina NextSeq. Sample preparations, AOI mask creation, and quantification is described in detail in the supplementary Methods. 


\section{Bioinformatics and Statistical Analysis}

For RNA-sequencing data, expression analysis was performed using Limma-voom(45) and pathway analysis was performed using Gene Set Enrichment Analysis(32). Reference gene sets were obtained from the Molecular Signatures Databases (MSigDB) (32), and fisher's exact test was used to test for the significance of the difference between the enriched genes and all the genes in the ISH panel for each of the signatures evaluated.

We defined the molecular subtype of "basal" vs "luminal" from the RNA-sequencing data using a previously published signature(9). Samples were classified into PPAR $\gamma^{\text {low }}$ and PPAR $\gamma^{\text {high }}$ groups based on the median mRNA expression of PPAR $\gamma$ based on NanoString counts, after normalization. After normalization of the IO360 expression data, previously described signatures $(21,22,46)$ were calculated across all samples. Briefly, expression signatures were calculated for each sample the using a linear model such that:

$$
S_{i}=\sum_{g=1}^{n}\left(\beta_{g} E_{g i}\right)+c_{S}
$$

Where the signature score, $S$, for sample, $i$, is the sum of the expression of each gene for a sample, $E_{g i}$, multiplied by predefined weights for each signature gene, $\beta_{g}$, and a constant for each signature, $c_{S}$. Scores were calculated and reported in $\log _{2}$ transformed expression space.

Correlation between analytes were calculated using either Spearman correlation coefficient $(\rho)$ or the Pearson correlation coefficient $\left(\mathrm{R}^{2}\right)$ as appropriate. Unsupervised hierarchical clustering was performed using complete linkage and Euclidean distance for clustering Z-scores of expressions of a given probe or analyte.

Differential protein expression from GeoMx data between phenotypes of interest was calculated using a mixed effect model allowing for random intercepts and slopes described as:

$$
\begin{gathered}
E_{g i}=\beta_{0}+S_{0 s}+\left(\beta_{1}+S_{1 s}\right) P_{1}+e_{s i x} \\
\left(S_{0 s}, S_{1 s}\right) \sim N\left(0,\left[\begin{array}{cc}
\tau_{00}^{2} & \rho \tau_{00} \tau_{11} \\
\rho \tau_{00} \tau_{11} & \tau_{11}^{2}
\end{array}\right]\right) x \\
e_{s i} \sim N\left(0, \sigma^{2}\right)
\end{gathered}
$$

Where the protein expression of a probe, $E_{s i}$, is tested against a phenotype, $P_{i}$ (e.g. AOI compartment), allowing for random intercepts and slopes modeling patient sample, $S$, to account for multiple AOIs tested within a patient slide. P-values for the mixed effect models were calculated using Satterthwaite's approximation for degrees of freedom(47-49).

P-values from statistical models were adjusted for multiple testing using the BenjaminiHochberg false-discovery rate (FDR)(50).

\section{Supplementary Materials}

Materials and Methods

Table S1. Clinical and molecular characteristics of MIBC cohort samples.

Table S2. RNA-ISH AOI characteristics and QC metrics.

Table S3. Spatial differential expression and compartment interaction analysis 
Data File S1. GeoMx Antibody data used in analysis

Data File S2. GeoMx RNA-ISH data used in analysis

Data File S3. Bulk expression signature analysis

Fig. S1. ROI placement on guide IF images

Fig. S2. QC of GeoMx profiling

Fig. S3. Relationship between bulk and GeoMx expression

Fig. S4. IHC quantification of CD8 and GZMB

Fig. S5. AOI expression of CD3 antibody

\section{References and Notes:}

1. H. B. Grossman, R. B. Natale, C. M. Tangen, V. O. Speights, N. J. Vogelzang, D. L. Trump, R. W. deVere White, M. F. Sarosdy, D. P. Wood, Jr., D. Raghavan, E. D. Crawford, Neoadjuvant chemotherapy plus cystectomy compared with cystectomy alone for locally advanced bladder cancer. $N$ Engl J Med 349, 859866 (2003).

2. J. Schardt, B. Roth, R. Seiler, Forty years of cisplatin-based chemotherapy in muscle-invasive bladder cancer: are we understanding how, who and when? World J Urol, (2018).

3. H. Zargar, P. N. Espiritu, A. S. Fairey, L. S. Mertens, C. P. Dinney, M. C. Mir, L. M. Krabbe, M. S. Cookson, N. E. Jacobsen, N. M. Gandhi, J. Griffin, J. S. Montgomery, N. Vasdev, E. Y. Yu, D. Youssef, E. Xylinas, N. J. Campain, W. Kassouf, M. A. Dall'Era, J. A. Seah, C. E. Ercole, S. Horenblas, S. S. Sridhar, J. S. McGrath, J. Aning, S. F. Shariat, J. L. Wright, A. C. Thorpe, T. M. Morgan, J. M. Holzbeierlein, T. J. Bivalacqua, S. North, D. A. Barocas, Y. Lotan, J. A. Garcia, A. J. Stephenson, J. B. Shah, B. W. van Rhijn, S. Daneshmand, P. E. Spiess, P. C. Black, Multicenter assessment of neoadjuvant chemotherapy for muscle-invasive bladder cancer. Eur Urol 67, 241-249 (2015).

4. T. International Collaboration of, P. Medical Research Council Advanced Bladder Cancer Working, R. European Organisation for, G. Treatment of Cancer Genito-Urinary Tract Cancer, G. Australian Bladder Cancer Study, G. National Cancer Institute of Canada Clinical Trials, Finnbladder, G. Norwegian Bladder Cancer Study, G. Club Urologico Espanol de Tratamiento Oncologico, G. Griffiths, R. Hall, R. Sylvester, D. Raghavan, M. K. Parmar, International phase III trial assessing neoadjuvant cisplatin, methotrexate, and vinblastine chemotherapy for muscle-invasive bladder cancer: long-term results of the BA06 30894 trial. $J$ Clin Oncol 29, 2171-2177 (2011).

5. B. Dietrich, S. Srinivas, Urothelial carcinoma: the evolving landscape of immunotherapy for patients with advanced disease. Res Rep Urol 10, 7-16 (2018).

6. M. Korpal, X. Puyang, Z. Jeremy Wu, R. Seiler, C. Furman, H. Z. Oo, M. Seiler, S. Irwin, V. Subramanian, J. Julie Joshi, C. K. Wang, V. Rimkunas, D. Tortora, H. Yang, N. Kumar, G. Kuznetsov, M. Matijevic, J. Chow, P. Kumar, J. Zou, J. Feala, L. Corson, R. Henry, A. Selvaraj, A. Davis, K. Bloudoff, J. Douglas, B. Kiss, M. Roberts, L. Fazli, P. C. Black, P. Fekkes, P. G. Smith, M. Warmuth, L. Yu, M. H. Hao, N. Larsen, M. Daugaard, P. Zhu, Evasion of immunosurveillance by genomic alterations of PPARgamma/RXRalpha in bladder cancer. Nat Commun 8, 103 (2017).

7. G. M. Balar AV, Rosenberg JE, Powles T, Petrylak DP, Bellmunt J, Loriot Y, Necchi A, Hoffman-Censits J, Perez-Gracia JL, Dawson NA, van der Heijden MS, Dreicer R, Srinivas S, Retz MM, Joseph RW, Drakaki A, Vaishampayan UN, Sridhar SS, Quinn DI, Durán I, Shaffer DR, Eigl BJ, Grivas PD, Yu EY, Li S, Kadel EE 3rd, Boyd Z, Bourgon R, Hegde PS, Mariathasan S, Thåström A, Abidoye OO, Fine GD, Bajorin DF, IMvigor210 Study Group., Atezolizumab as first-line treatment in cisplatin-ineligible patients with locally advanced and metastatic urothelial carcinoma: a single-arm, multicentre, phase 2 trial. Lancet 389, 67-76 (2017).

8. J. S. Damrauer, K. A. Hoadley, D. D. Chism, C. Fan, C. J. Tiganelli, S. E. Wobker, J. J. Yeh, M. I. Milowsky, G. Iyer, J. S. Parker, W. Y. Kim, Intrinsic subtypes of high-grade bladder cancer reflect the hallmarks of breast cancer biology. Proc Natl Acad Sci U S A 111, 3110-3115 (2014). 
9. $\quad$ W. Choi, S. Porten, S. Kim, D. Willis, E. R. Plimack, J. Hoffman-Censits, B. Roth, T. Cheng, M. Tran, I. L. Lee, J. Melquist, J. Bondaruk, T. Majewski, S. Zhang, S. Pretzsch, K. Baggerly, A. Siefker-Radtke, B. Czerniak, C. P. Dinney, D. J. McConkey, Identification of distinct basal and luminal subtypes of muscleinvasive bladder cancer with different sensitivities to frontline chemotherapy. Cancer Cell 25, 152-165 (2014).

10. D. J. McConkey, W. Choi, Y. Shen, I. L. Lee, S. Porten, S. F. Matin, A. M. Kamat, P. Corn, R. E. Millikan, C. Dinney, B. Czerniak, A. O. Siefker-Radtke, A Prognostic Gene Expression Signature in the Molecular Classification of Chemotherapy-naive Urothelial Cancer is Predictive of Clinical Outcomes from Neoadjuvant Chemotherapy: A Phase 2 Trial of Dose-dense Methotrexate, Vinblastine, Doxorubicin, and Cisplatin with Bevacizumab in Urothelial Cancer. Eur Urol 69, 855-862 (2016).

11. R. Seiler, E. A. Gibb, N. Q. Wang, H. Z. Oo, H. M. Lam, K. E. van Kessel, C. S. Voskuilen, B. Winters, N. Erho, M. M. Takhar, J. Douglas, F. Vakar-Lopez, S. J. Crabb, B. W. G. van Rhijn, E. E. Fransen van de Putte, E. C. Zwarthoff, G. N. Thalmann, E. Davicioni, J. L. Boormans, M. Dall'Era, M. S. van der Heijden, J. L. Wright, P. C. Black, Divergent Biological Response to Neoadjuvant Chemotherapy in Muscleinvasive Bladder Cancer. Clin Cancer Res, (2018).

12. R. Saito, C. C. Smith, T. Utsumi, L. M. Bixby, J. Kardos, S. E. Wobker, K. G. Stewart, S. Chai, U. Manocha, K. M. Byrd, J. S. Damrauer, S. E. Williams, B. G. Vincent, W. Y. Kim, Molecular SubtypeSpecific Immunocompetent Models of High-Grade Urothelial Carcinoma Reveal Differential Neoantigen Expression and Response to Immunotherapy. Cancer Res 78, 3954-3968 (2018).

13. V. Stuhler, J. M. Maas, J. Bochem, I. A. da Costa, T. Todenhofer, A. Stenzl, J. Bedke, Molecular predictors of response to PD-1/PD-L1 inhibition in urothelial cancer. World J Urol, (2018).

14. H.-C. J. Rosenberg JE, Powles T, van der Heijden MS, Balar AV, Necchi A, Dawson N, O'Donnell PH, Balmanoukian A, Loriot Y, Srinivas S, Retz MM, Grivas P, Joseph RW, Galsky MD, Fleming MT, Petrylak DP, Perez-Gracia JL, Burris HA, Castellano D, Canil C, Bellmunt J, Bajorin D, Nickles D, Bourgon R, Frampton GM, Cui N, Mariathasan S, Abidoye O, Fine GD, Dreicer R, Atezolizumab in patients with locally advanced and metastatic urothelial carcinoma who have progressed following treatment with platinum-based chemotherapy: a single-arm, multicentre, phase 2 trial. Lancet 387, 19091920 (2016).

15. P. Sharma, M. Retz, A. Siefker-Radtke, A. Baron, A. Necchi, J. Bedke, E. R. Plimack, D. Vaena, M. O. Grimm, S. Bracarda, J. A. Arranz, S. Pal, C. Ohyama, A. Saci, X. Qu, A. Lambert, S. Krishnan, A. Azrilevich, M. D. Galsky, Nivolumab in metastatic urothelial carcinoma after platinum therapy (CheckMate 275): a multicentre, single-arm, phase 2 trial. Lancet Oncol 18, 312-322 (2017).

16. J. Kardos, S. Chai, L. E. Mose, S. R. Selitsky, B. Krishnan, R. Saito, M. D. Iglesia, M. I. Milowsky, J. S. Parker, W. Y. Kim, B. G. Vincent, Claudin-low bladder tumors are immune infiltrated and actively immune suppressed. JCI Insight 1, e85902 (2016).

17. P. M. Forde, J. E. Chaft, K. N. Smith, V. Anagnostou, T. R. Cottrell, M. D. Hellmann, M. Zahurak, S. C. Yang, D. R. Jones, S. Broderick, R. J. Battafarano, M. J. Velez, N. Rekhtman, Z. Olah, J. Naidoo, K. A. Marrone, F. Verde, H. Guo, J. Zhang, J. X. Caushi, H. Y. Chan, J. W. Sidhom, R. B. Scharpf, J. White, E. Gabrielson, H. Wang, G. L. Rosner, V. Rusch, J. D. Wolchok, T. Merghoub, J. M. Taube, V. E.

Velculescu, S. L. Topalian, J. R. Brahmer, D. M. Pardoll, Neoadjuvant PD-1 Blockade in Resectable Lung Cancer. N Engl J Med 378, 1976-1986 (2018).

18. P. C. Tumeh, C. L. Harview, J. H. Yearley, I. P. Shintaku, E. J. Taylor, L. Robert, B. Chmielowski, M. Spasic, G. Henry, V. Ciobanu, A. N. West, M. Carmona, C. Kivork, E. Seja, G. Cherry, A. J. Gutierrez, T. R. Grogan, C. Mateus, G. Tomasic, J. A. Glaspy, R. O. Emerson, H. Robins, R. H. Pierce, D. A. Elashoff, C. Robert, A. Ribas, PD-1 blockade induces responses by inhibiting adaptive immune resistance. Nature 515, 568-571 (2014).

19. F. R. Hirsch, A. McElhinny, D. Stanforth, J. Ranger-Moore, M. Jansson, K. Kulangara, W. Richardson, P. Towne, D. Hanks, B. Vennapusa, A. Mistry, R. Kalamegham, S. Averbuch, J. Novotny, E. Rubin, K. Emancipator, I. McCaffery, J. A. Williams, J. Walker, J. Longshore, M. S. Tsao, K. M. Kerr, PD-L1 Immunohistochemistry Assays for Lung Cancer: Results from Phase 1 of the Blueprint PD-L1 IHC Assay Comparison Project. J Thorac Oncol 12, 208-222 (2017).

20. D. L. Rimm, G. Han, J. M. Taube, E. S. Yi, J. A. Bridge, D. B. Flieder, R. Homer, W. W. West, H. Wu, A. C. Roden, J. Fujimoto, H. Yu, R. Anders, A. Kowalewski, C. Rivard, J. Rehman, C. Batenchuk, V. Burns, F. R. Hirsch, Wistuba, II, A Prospective, Multi-institutional, Pathologist-Based Assessment of 4 Immunohistochemistry Assays for PD-L1 Expression in Non-Small Cell Lung Cancer. JAMA Oncol 3, 1051-1058 (2017). 
21. M. Ayers, J. Lunceford, M. Nebozhyn, E. Murphy, A. Loboda, D. R. Kaufman, A. Albright, J. D. Cheng, S. P. Kang, V. Shankaran, S. A. Piha-Paul, J. Yearley, T. Y. Seiwert, A. Ribas, T. K. McClanahan, IFNgamma-related mRNA profile predicts clinical response to PD-1 blockade. J Clin Invest 127, 2930-2940 (2017).

22. P. Danaher, S. Warren, R. Lu, J. Samayoa, A. Sullivan, I. Pekker, B. Wallden, F. M. Marincola, A. Cesano, Pan-cancer adaptive immune resistance as defined by the Tumor Inflammation Signature (TIS): results from The Cancer Genome Atlas (TCGA). J Immunother Cancer 6, 63 (2018).

23. R. Cristescu, R. Mogg, M. Ayers, A. Albright, E. Murphy, J. Yearley, X. Sher, X. Q. Liu, H. Lu, M. Nebozhyn, C. Zhang, J. K. Lunceford, A. Joe, J. Cheng, A. L. Webber, N. Ibrahim, E. R. Plimack, P. A. Ott, T. Y. Seiwert, A. Ribas, T. K. McClanahan, J. E. Tomassini, A. Loboda, D. Kaufman, Pan-tumor genomic biomarkers for PD-1 checkpoint blockade-based immunotherapy. Science 362, (2018).

24. J. Galon, B. A. Fox, C. B. Bifulco, G. Masucci, T. Rau, G. Botti, F. M. Marincola, G. Ciliberto, F. Pages, P. A. Ascierto, M. Capone, Immunoscore and Immunoprofiling in cancer: an update from the melanoma and immunotherapy bridge 2015. J Transl Med 14, 273 (2016).

25. G. Bindea, B. Mlecnik, M. Tosolini, A. Kirilovsky, M. Waldner, A. C. Obenauf, H. Angell, T. Fredriksen, L. Lafontaine, A. Berger, P. Bruneval, W. H. Fridman, C. Becker, F. Pages, M. R. Speicher, Z. Trajanoski, J. Galon, Spatiotemporal dynamics of intratumoral immune cells reveal the immune landscape in human cancer. Immunity 39, 782-795 (2013).

26. S. G. Rodriques, R. R. Stickels, A. Goeva, C. A. Martin, E. Murray, C. R. Vanderburg, J. Welch, L. M. Chen, F. Chen, E. Z. Macosko, Slide-seq: A scalable technology for measuring genome-wide expression at high spatial resolution. Science 363, 1463-1467 (2019).

27. P. L. Stahl, F. Salmen, S. Vickovic, A. Lundmark, J. F. Navarro, J. Magnusson, S. Giacomello, M. Asp, J. O. Westholm, M. Huss, A. Mollbrink, S. Linnarsson, S. Codeluppi, A. Borg, F. Ponten, P. I. Costea, P. Sahlen, J. Mulder, O. Bergmann, J. Lundeberg, J. Frisen, Visualization and analysis of gene expression in tissue sections by spatial transcriptomics. Science 353, 78-82 (2016).

28. G. Wang, J. R. Moffitt, X. Zhuang, Multiplexed imaging of high-density libraries of RNAs with MERFISH and expansion microscopy. Sci Rep 8, 4847 (2018).

29. J. Decalf, M. L. Albert, J. Ziai, New tools for pathology: a user's review of a highly multiplexed method for in situ analysis of protein and RNA expression in tissue. J Pathol 247, 650-661 (2019).

30. M. I. Toki, C. R. Merritt, P. F. Wong, J. W. Smithy, H. M. Kluger, K. N. Syrigos, G. T. Ong, S. E. Warren, J. M. Beechem, D. L. Rimm, High-plex predictive marker discovery for melanoma immunotherapy treated patients using Digital Spatial Profiling. Clin Cancer Res, (2019).

31. D. Aran, M. Sirota, A. J. Butte, Systematic pan-cancer analysis of tumour purity. Nat Commun 6, 8971 (2015).

32. A. Subramanian, P. Tamayo, V. K. Mootha, S. Mukherjee, B. L. Ebert, M. A. Gillette, A. Paulovich, S. L. Pomeroy, T. R. Golub, E. S. Lander, J. P. Mesirov, Gene set enrichment analysis: a knowledge-based approach for interpreting genome-wide expression profiles. Proc Natl Acad Sci U S A 102, 15545-15550 (2005).

33. M. F. Rogers, H. A. Shihab, T. R. Gaunt, C. Campbell, CScape: a tool for predicting oncogenic singlepoint mutations in the cancer genome. Sci Rep 7, 11597 (2017).

34. S. Mariathasan, S. J. Turley, D. Nickles, A. Castiglioni, K. Yuen, Y. Wang, E. E. Kadel, III, H. Koeppen, J. L. Astarita, R. Cubas, S. Jhunjhunwala, R. Banchereau, Y. Yang, Y. Guan, C. Chalouni, J. Ziai, Y. Senbabaoglu, S. Santoro, D. Sheinson, J. Hung, J. M. Giltnane, A. A. Pierce, K. Mesh, S. Lianoglou, J. Riegler, R. A. D. Carano, P. Eriksson, M. Hoglund, L. Somarriba, D. L. Halligan, M. S. van der Heijden, Y. Loriot, J. E. Rosenberg, L. Fong, I. Mellman, D. S. Chen, M. Green, C. Derleth, G. D. Fine, P. S. Hegde, R. Bourgon, T. Powles, TGFbeta attenuates tumour response to PD-L1 blockade by contributing to exclusion of T cells. Nature 554, 544-548 (2018).

35. M. B. H. Thomsen, I. Nordentoft, P. Lamy, S. Vang, L. Reinert, C. K. Mapendano, S. Hoyer, T. F. Orntoft, J. B. Jensen, L. Dyrskjot, Comprehensive multiregional analysis of molecular heterogeneity in bladder cancer. Sci Rep 7, 11702 (2017).

36. J. I. Warrick, G. Sjodahl, M. Kaag, J. D. Raman, S. Merrill, L. Shuman, G. Chen, V. Walter, D. J. DeGraff, Intratumoral Heterogeneity of Bladder Cancer by Molecular Subtypes and Histologic Variants. Eur Urol 75, 18-22 (2019).

37. S. Spranger, R. Bao, T. F. Gajewski, Melanoma-intrinsic beta-catenin signalling prevents anti-tumour immunity. Nature 523, 231-235 (2015). 
38. B. Chen, M. S. Khodadoust, C. L. Liu, A. M. Newman, A. A. Alizadeh, Profiling Tumor Infiltrating Immune Cells with CIBERSORT. Methods Mol Biol 1711, 243-259 (2018).

39. A. M. Newman, C. L. Liu, M. R. Green, A. J. Gentles, W. Feng, Y. Xu, C. D. Hoang, M. Diehn, A. A. Alizadeh, Robust enumeration of cell subsets from tissue expression profiles. Nat Methods 12, 453-457 (2015).

40. B. Li, E. Severson, J. C. Pignon, H. Zhao, T. Li, J. Novak, P. Jiang, H. Shen, J. C. Aster, S. Rodig, S. Signoretti, J. S. Liu, X. S. Liu, Comprehensive analyses of tumor immunity: implications for cancer immunotherapy. Genome Biol 17, 174 (2016).

41. X. Wang, J. Park, K. Susztak, N. R. Zhang, M. Li, Bulk tissue cell type deconvolution with multi-subject single-cell expression reference. Nat Commun 10, 380 (2019).

42. V. Svensson, S. A. Teichmann, O. Stegle, SpatialDE: identification of spatially variable genes. Nat Methods 15, 343-346 (2018).

43. A. Dobin, C. A. Davis, F. Schlesinger, J. Drenkow, C. Zaleski, S. Jha, P. Batut, M. Chaisson, T. R. Gingeras, STAR: ultrafast universal RNA-seq aligner. Bioinformatics 29, 15-21 (2013).

44. N. L. Bray, H. Pimentel, P. Melsted, L. Pachter, Near-optimal probabilistic RNA-seq quantification. Nat Biotechnol 34, 525-527 (2016).

45. C. W. Law, Y. Chen, W. Shi, G. K. Smyth, voom: Precision weights unlock linear model analysis tools for RNA-seq read counts. Genome Biol 15, R29 (2014).

46. P. Danaher, S. Warren, L. Dennis, L. D'Amico, A. White, M. L. Disis, M. A. Geller, K. Odunsi, J. Beechem, S. P. Fling, Gene expression markers of Tumor Infiltrating Leukocytes. J Immunother Cancer 5, 18 (2017).

47. A. C. Fai, PL Approximate F-Tests of Multiple Degree of Freedom Hypotheses in Generalised Least Squares Analyses of Unbalanced Split-Plot Experiments. Journal of Statistical Computation and Simulation 54, 363-378 (1996).

48. F. B. Giesbrecht, JC, Two-Stage Analysis Based on a Mixed Model: Large-Sample Asymptotic Theory and Small-Sample Simulation Results. Biometrics 41, 477-486 (1985).

49. A. B. Kuznetsova, PB; Christensen RHB, lmerTest Package: Tests in Linear Mixed Effects Models. J Stat Software 82, (2017).

50. Y. H. Benjamini, Y, Controlling the False Discovery Rate: A Practical and Powerful Approach to Multiple Testing. Journal of the Royal Statistical Society 57, 289-300 (1995).

Acknowledgments: We thank Alessandra Cusano, Lihua Yu, and Ping Zhu for helping review the manuscript. Funding: This study was funded by H3 Biomedicine and NanoString Inc., Author contributions: JWR, ZZ performed data analysis and prepared the manuscript; DMZ, JG coordinated and ran GeoMx profiling; ZKN created and ran the RNA-ISH sequencing pipeline; SD, MK, PK, VR procured FFPE samples and coordinated mutation profiling, RNA sequencing, and IHC profiling; SW, MH, JB, PK, VR conceived of study design and managed study execution Competing interests: JWR, ZKN, DMZ, JG, YL, SW, MH, JB are employees of and hold stock in NanoString Inc, which is the manufacturer of the nCounter and GeoMx platforms described herein. GeoMx is available for research use only (RUO) purposes. ZZ, SD, MK, PK, VR are employees of H3 Biomedicine Inc., which is currently developing a PPARG inhibitor. Data and materials availability: Processed data files are provided as supplemental material. Original nCounter expression files have been uploaded to GEO, and RNA sequencing files have been uploaded to the SRA. High resolution image files may be made available through an MTA. 
Figures:

A

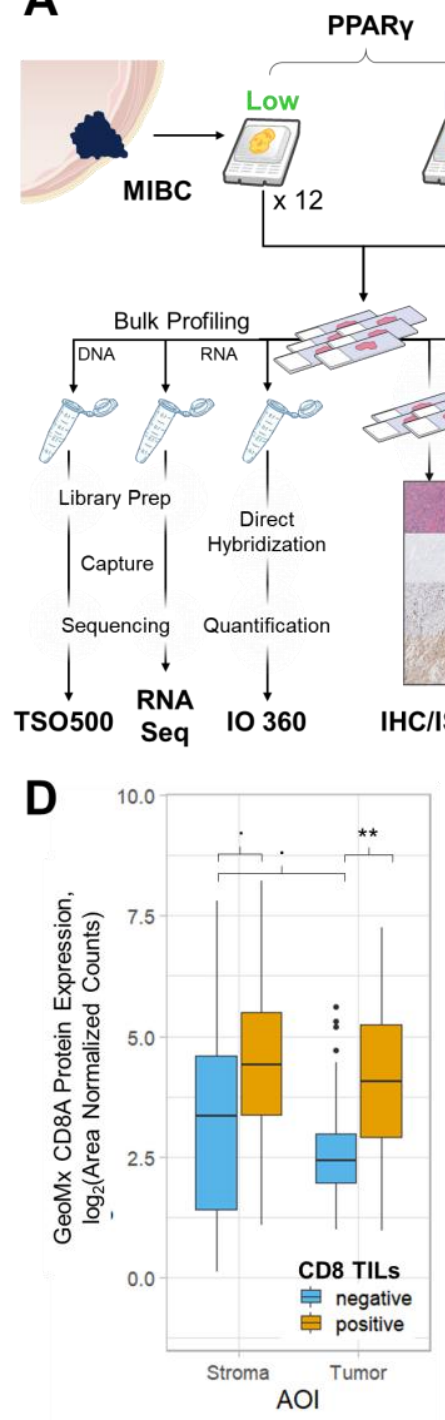

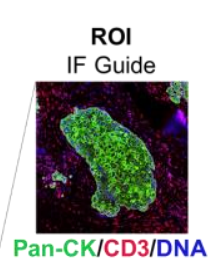

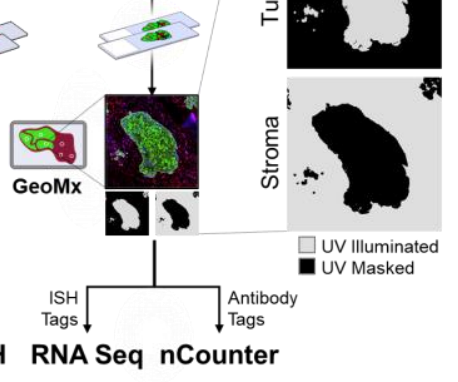

E

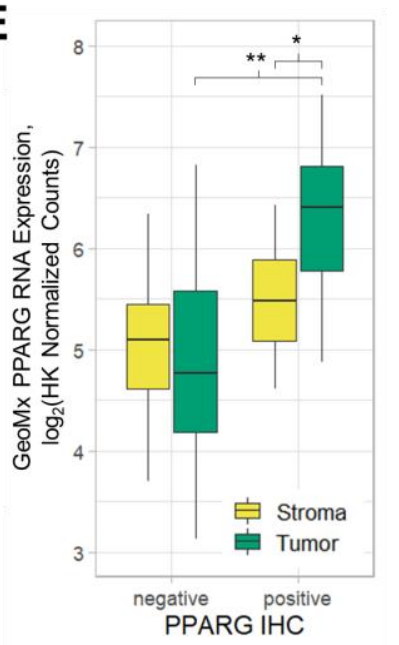

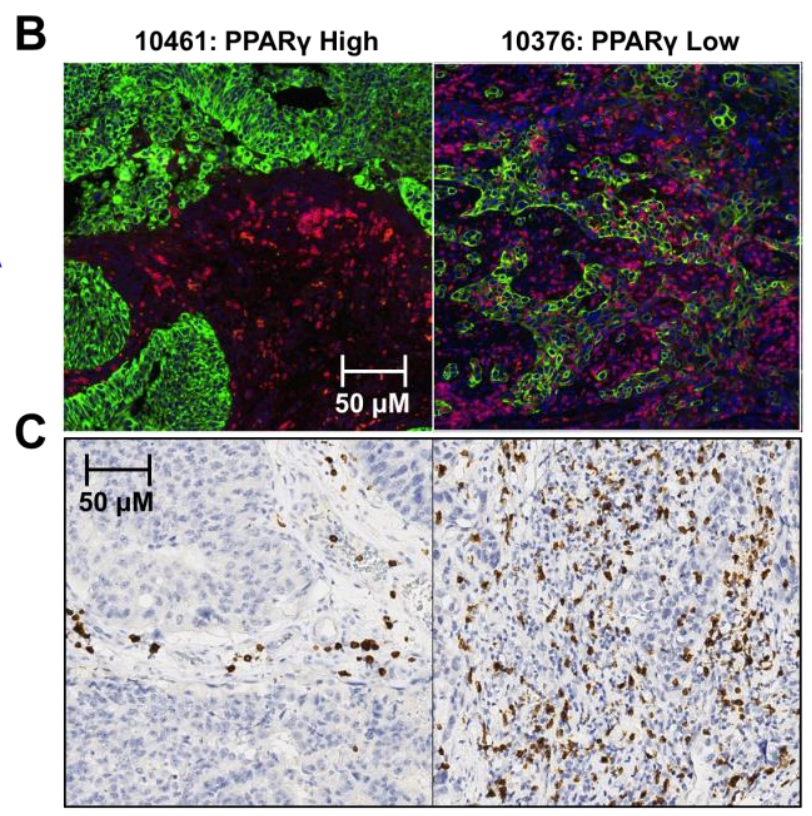

C

$\mathbf{F}$

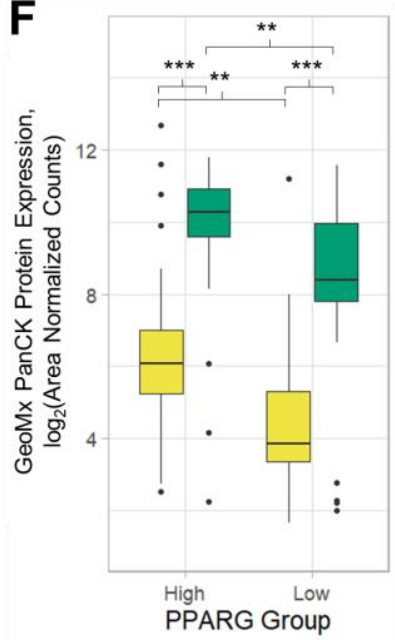

Fig. 1. Study overview and qualification of GeoMx readouts (A) Patient samples analyzed using separate workflows for bulk and spatial profiling. Samples were prepared for multiple workstreams for bulk of DNA and RNA and spatial profiling of RNA and protein expression. GeoMx analysis involves selection of regions of interest (ROIs) based on whole-slide IF and subsequent UV illumination of specific paths into areas of illumination (AOI, gray) based on Pan-CK staining by IF to collect oligo tags for downstream quantification. (B) Example IF guide images placed for selected ROIs during GeoMx workflow and (C) related CD8 IHC images of the same ROI for either a representative PPAR $\gamma^{\text {high }}$ patient (left) or PPAR $\gamma^{\text {low }}$ patient (right) (D-F) Example validation of concordance between GeoMx profiling and (D) IHC of CD8 TILs, (E) PPARG expression by ISH, or (F) between guide markers and related antibody probes 
(Pan-CK, left; CD3, right). Nominal significance shown for mixed effect model, denoted as . for $\mathrm{p}<0.1, * \mathrm{p}<0.05, * * \mathrm{p}<0.01, * * * \mathrm{p}<0.001$.

A

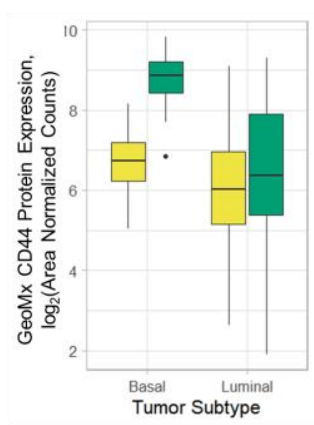

C

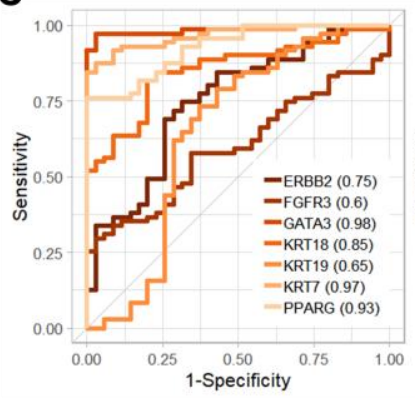

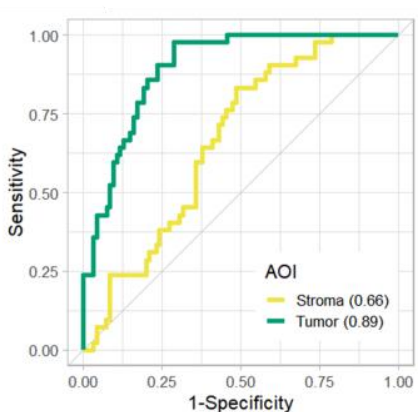

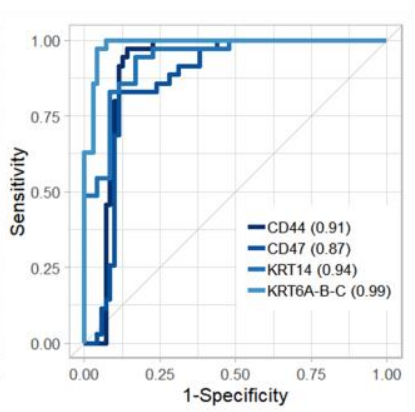

B

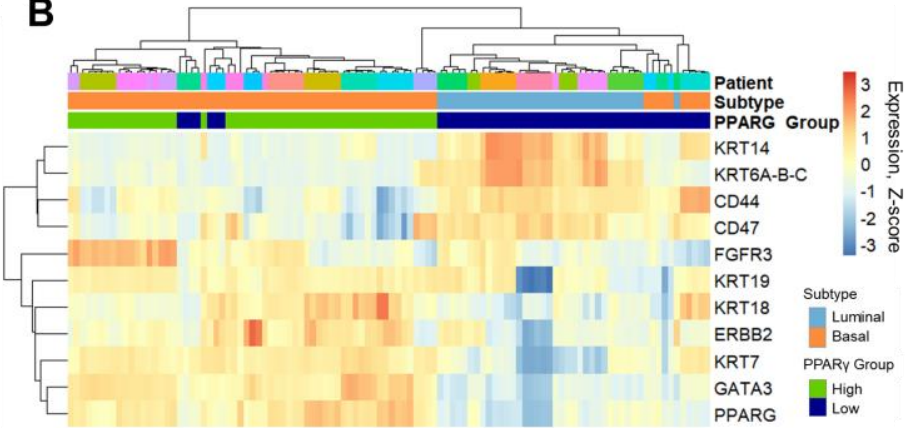

D

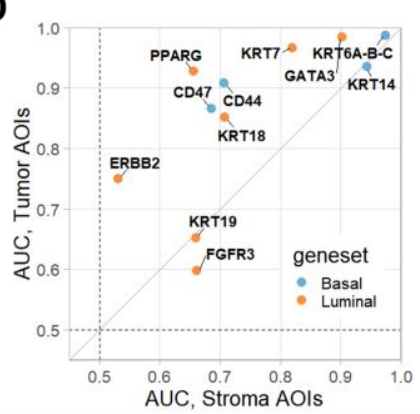

E

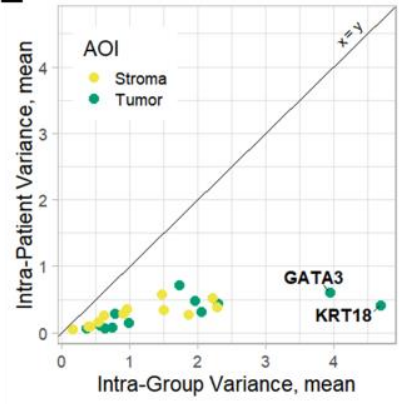

Fig. 2. Tumor intrinsic subtyping (A) Analysis of protein expression of CD44 as a marker for tumor subtype, per AOI. ROC curves shown for tumor and stromal AOIs analyzed separately. AUC values shown for each AOI type. (B) Unsupervised hierarchical clustering of subtyping probes included in RNA-ISH GeoMx analysis. Annotations show patient ID, RNA sequencing identified subtype (orange = luminal, light blue $=$ basal), and PPARG expression group (dark blue $=$ low, green $=$ high $)(\mathbf{C})$ ROC analysis of MIBC subtype classification for RNA-ISH markers measured in tumor AOIs. (D) Comparison of AUCs from ROC analysis of tumor or stromal AOIs from C. (E) Comparison of average variance within subtype or patient samples 

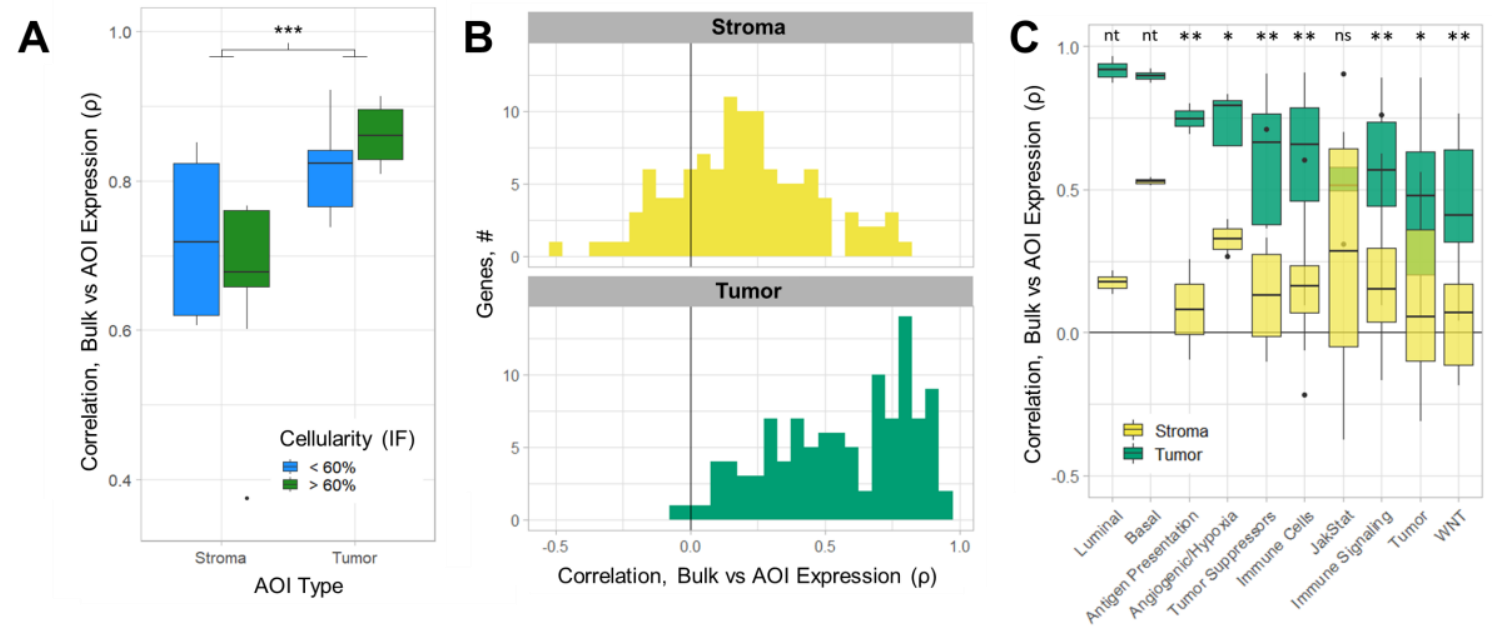

Fig 3. Bulk profiling masks expression patterns observed in the stroma (A) Correlation of gene expression observed in IO360 profiling and RNA-ISH GeoMx profiling. Correlations were calculated between bulk samples and each AOI from the same sample and then separated by AOI type and tumor cellularity estimated from the IF image used to select ROIs. Significantly higher ( $p<0.001$, Welch's t-test) correlation observed in tumor AOIs than Stromal AOIs. (B-C) Gene-based calculations of correlation based on average AOI expression for a patient within each AOI type, showing correlations across all genes based on (B) AOI location (Stroma, top; tumor, bottom) and (C) associated gene set information. Significance (p, Welch's t-test) indicated by $*<0.05, * *<0.01$, ns $>0.05$, nt for not tested when $n_{\text {genes }}<3$ 
A

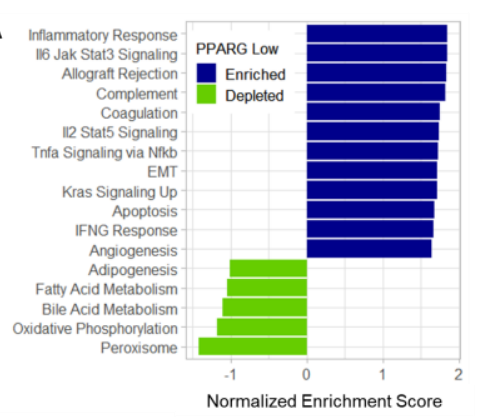

D
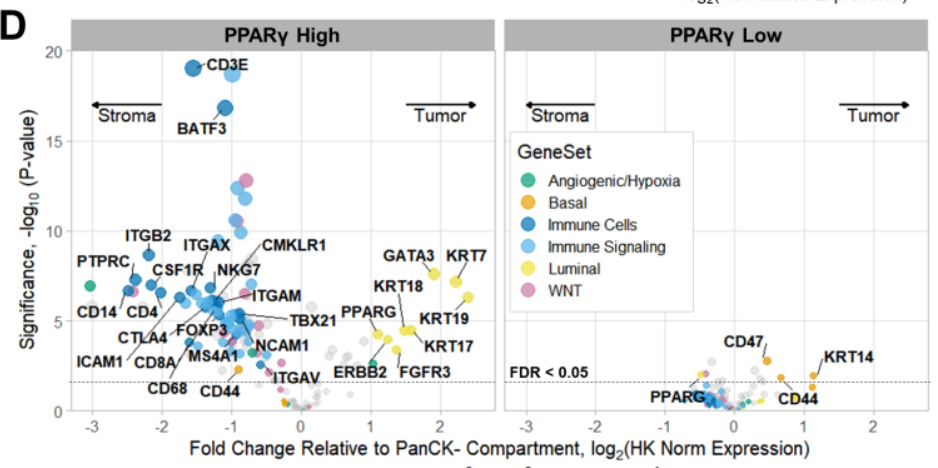

$\mathbf{E}$

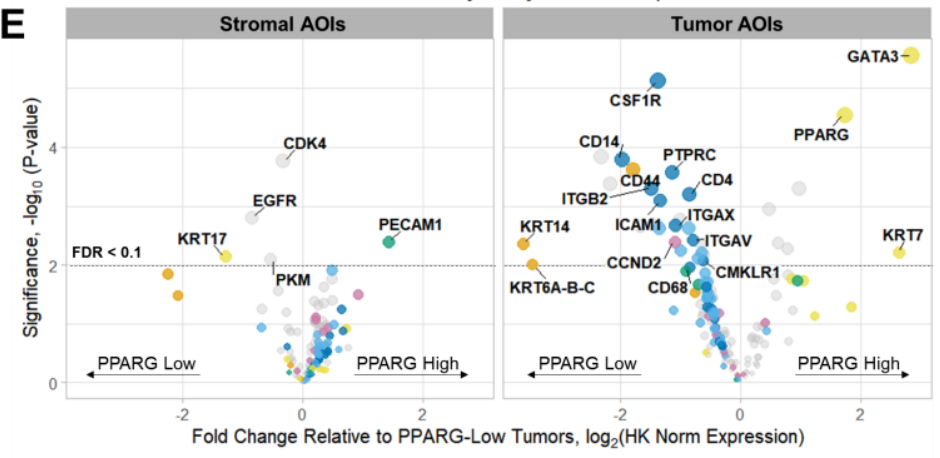

B
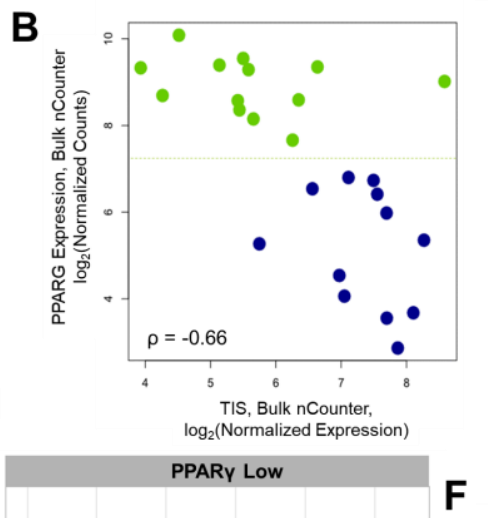

C

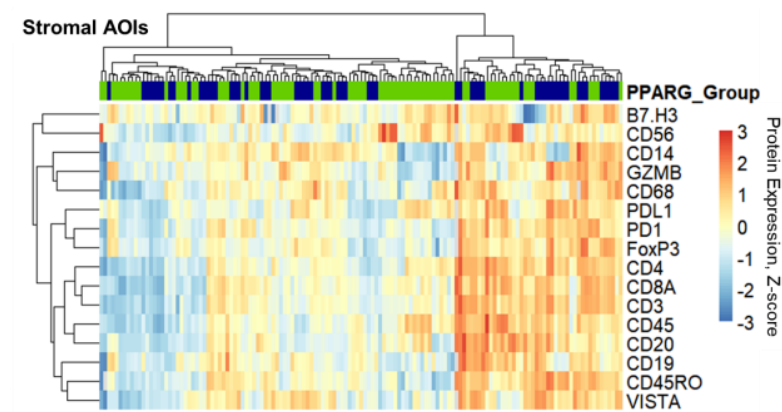

Tumor AOls

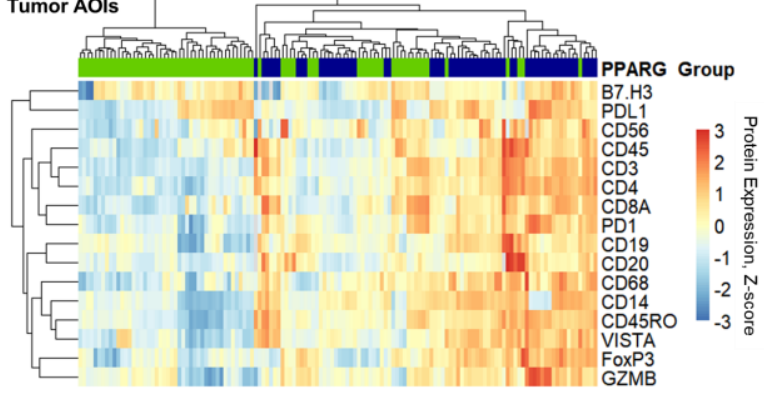

Fig 4. Phenotypic analysis of the tumor microenvironment in MIBC (A) Gene set enrichment analysis results from bulk RNA-sequencing analysis indicative of high immune-cell related signaling detection in PPAR $\gamma^{\text {low }}$ tumors (B) PPAR $\gamma$ expression level vs tumor inflammation signature (TIS), colored base to PPAR $\gamma$ status from IO360 analysis (high = green, low = dark blue) (C) IO360 Signatures score expression based on PPAR $\gamma$ status, binned by type of signature. Significance ( $\mathrm{p}$, Welch's t-test) indicated by $*<0.05, * *<$ $0.01, * * *<1-\mathrm{e} 3$ (D) Differential expression analysis GeoMx RNA-ISH data shows stromal enrichment of immune cell and immune signaling genes in PPAR $\gamma^{\text {high }}$ tumors (left), while no significant difference is observed in PPAR $\gamma^{\text {low }}$ tumors (right). Genes colored based on gene sets, labelled for immune cells or subtyping markers for FDR < 0.05 (D) Differential expression analysis GeoMx RNA-ISH data based on PPARG status in each type of AOI. Genes colored based on gene sets, labelled for immune cells or subtyping markers for FDR $<0.1$ in Tumor AOIs, all genes with FDR $<0.1$ labeled in stromal AOIs (F) USHC of immune cell markers from antibody analysis of either stromal AOIs (top) or tumor AOIs (bottom). 
A
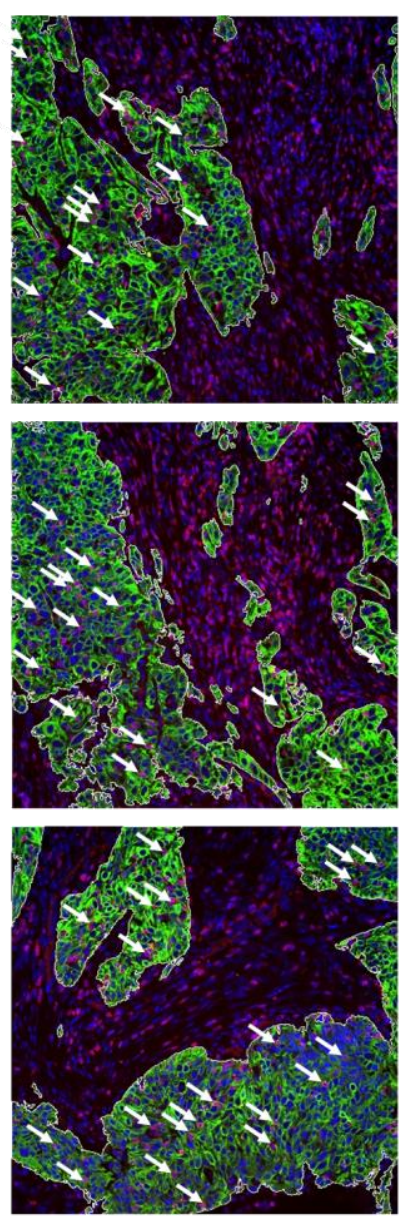

B

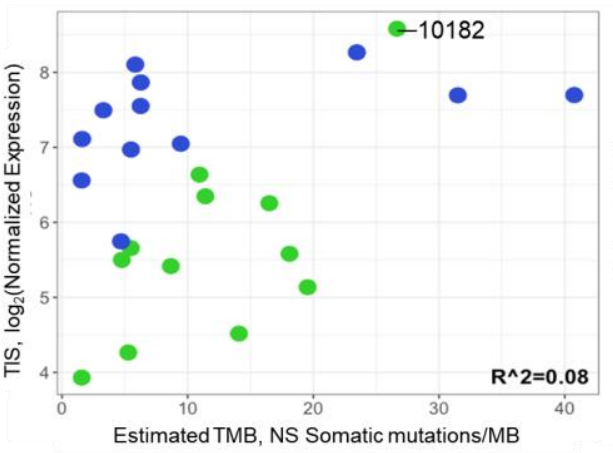

C
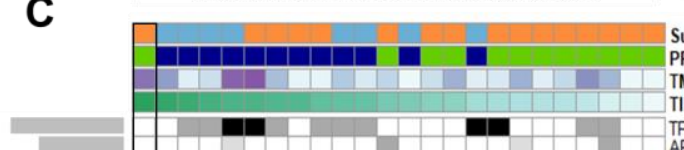

Subtype

MB
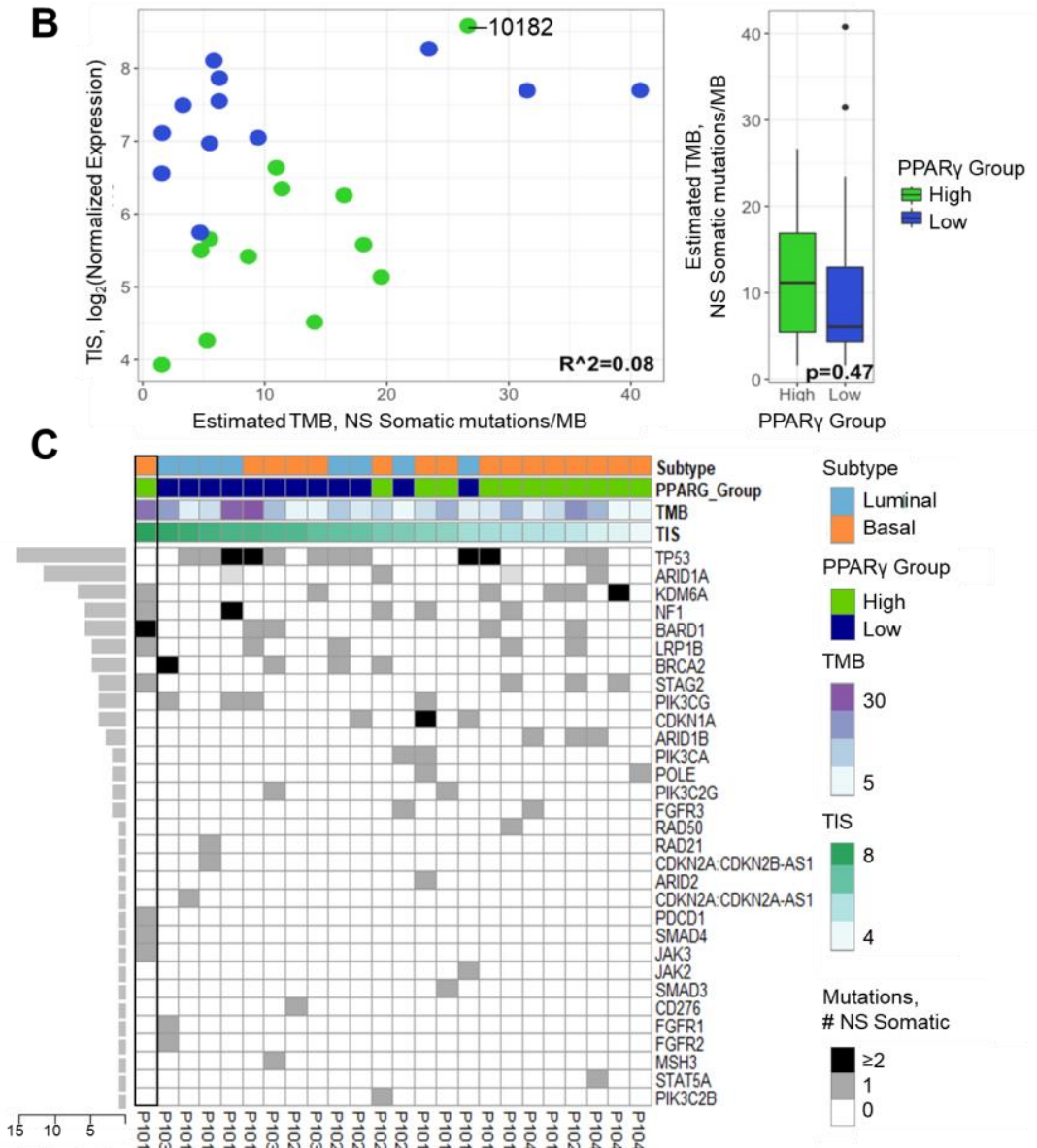

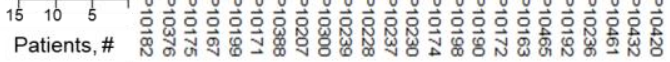

Fig 5. Tumor mutations associated with high TILs in patient 10182 (A) IF images of ROIs collected from sample 10182. Markers stained include Pan-CK (green), CD3 (red), and nuclear stain (blue). Arrows indicate CD3+ TILs within Pan-CK AOIs. (B) TMB analysis of bulk samples vs TIS or PPARG group, highlighting sample 10182 (C) Oncoprint of mutations across samples showing tumor-intrinsic subtype, PPAR $\gamma$ expression, TMB counts, and TIS. 

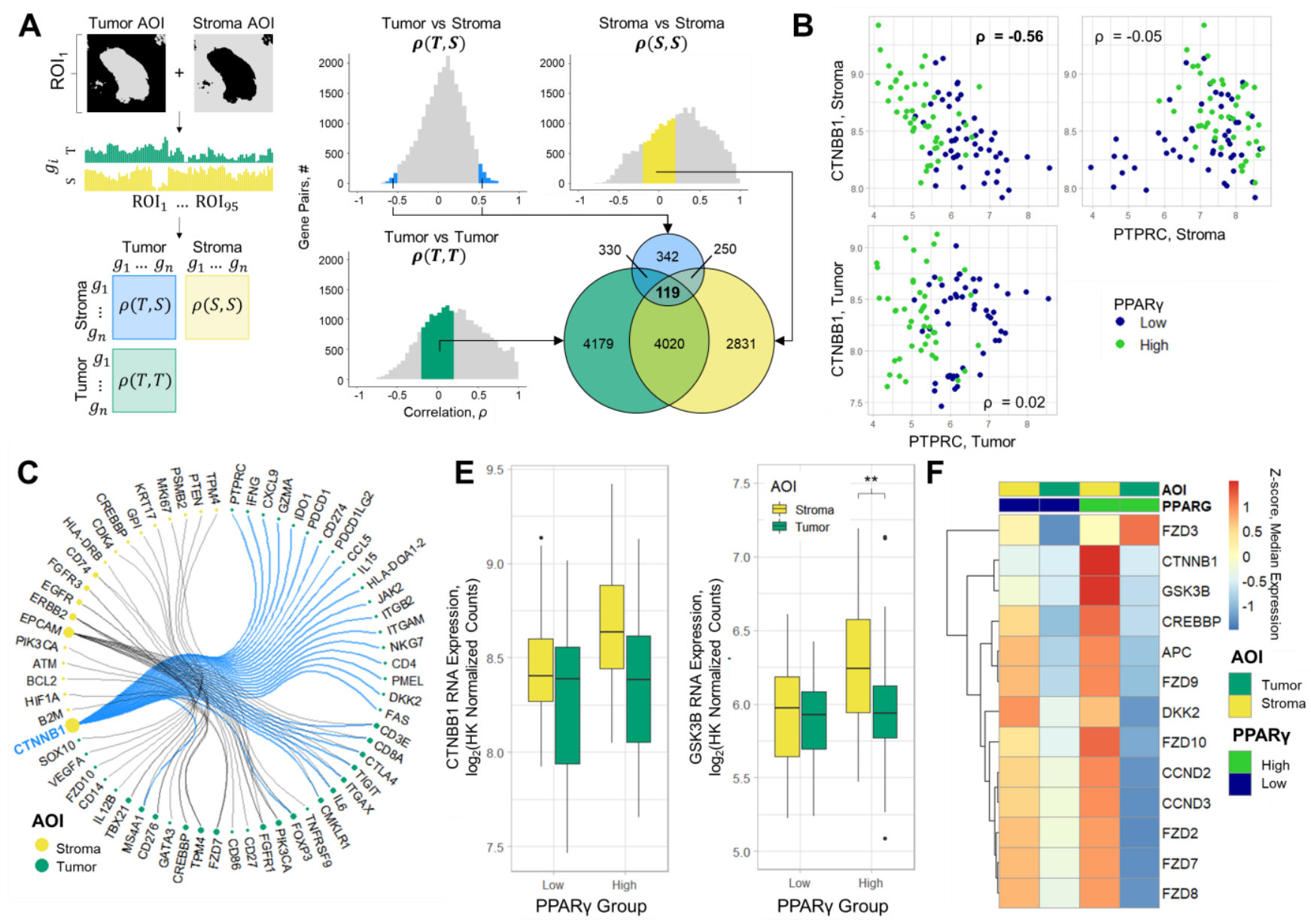

Fig 6. Spatial analysis identifies a stromal WNT- $\beta$-Catenin axis associated with immune cells (A) RNA-ISH tag expression from AOIs (gray areas, left) was used to calculate the correlation coefficient $(\rho$, Spearman) for expression values for all pairs of genes across different combinations of types of AOIs measured across 95 ROIs. Potential paracrine associations $(\mathrm{n}=119)$ were identified by selecting genes with high correlation between expression in tumor and stromal compartments $(|\rho(T, S)|>0.5)$, while also having minimal correlation within the same compartment type $(|\rho(T, T ; S, S)|<0.2)$. (C) Expression of CTNNB1 ( $\beta$-Catenin) and PTPRC (CD45) based on compartment of measurement, demonstrating the specific correlation between stromal $\beta$-catenin and tumor expression of PTPRC (D) Map of identified tumor-stroma interactions. Lines indicate tumor-stroma specific correlations, with pairs that include stromal CTNBB1 highlighted in blue. Nodes colored by AOI of expression, size based on number of interactions identified. (E) Expression of $\beta$-catenin and GSK3B based on PPAR $\gamma$ expression and AOI. ** FDR $<0.01$ (F) Heatmap of WNT/ $\beta$-catenin pathway members in each compartment based on PPAR $\gamma$ expression. 


\begin{tabular}{|c|c|c|c|}
\hline & & $\begin{array}{c}\text { PPAR } \gamma^{\text {high }} \\
(n=13)\end{array}$ & $\begin{array}{c}\text { PPAR } \gamma^{\text {low }} \\
(\mathrm{n}=12)\end{array}$ \\
\hline \multirow[t]{4}{*}{ Stage } & I & 2 & 0 \\
\hline & II & 3 & 0 \\
\hline & III & 5 & 5 \\
\hline & IV & 3 & 7 \\
\hline Age* & & $58(44-75)$ & $67(43-72)$ \\
\hline \multirow[t]{2}{*}{ LN Status } & Negative & 10 & 6 \\
\hline & Positive & 3 & 6 \\
\hline \multirow[t]{2}{*}{ Gender } & Female & 1 & 3 \\
\hline & Male & 12 & 9 \\
\hline Genomic & Basal & 0 & 8 \\
\hline Subtype & Luminal & 13 & 4 \\
\hline \multirow[t]{3}{*}{ CD8 TILS } & $<1 \%$ & 9 & 3 \\
\hline & $>1 \% \&<5 \%$ & 3 & 4 \\
\hline & $>5 \%$ & 1 & 5 \\
\hline Tumor & $<60 \%$ & 4 & 7 \\
\hline Cellularity $^{\dagger}$ & $>60 \%$ & 9 & 2 \\
\hline GeoMx & Antibody & 13 & 10 \\
\hline Profiling & RNA-ISH & 10 & 9 \\
\hline
\end{tabular}

Table 1. Clinical, genomic, and TME characteristics of MIBC cohort. *median age and range shown. 'Estimated from RNA-ISH IF Pan-CK staining 


\section{Supplementary Methods.}

\section{Sample preparation and readout of protein Digital Spatial Profiling}

For multiplex antibody analysis, a cocktail of 40 primary antibodies (Supplementary Data File 1), each with a unique, UV photocleavable indexing oligo, and 2 fluorescent markers (Pan$\mathrm{CK}$ and CD3) were added to a $5 \mu \mathrm{m}$ FFPE slide at $4{ }^{\circ} \mathrm{C}$ overnight. Pan-CK and CD3 were used to identify tumor cells and T cells respectively. Syto83 (nuclear DNA stain) was added to the FFPE tissues for 5 minutes at room temperature. After washing off the extra antibodies in TBST, the tissue slides were placed on the stage of an inverted microscope. A custom gasket was then clamped onto each slide, allowing the tissue to be submerged in $1.5 \mathrm{~mL}$ of buffer solution. Under the microscope, wide field fluorescence imaging was performed with epi-illumination from visible LED light engine. 20x images were stitched together to yield a high-resolution image of the tissue area of interest. Two of the 25 samples (10171 and 10230) were omitted from further processing due to poor tissue attachment. Of the remaining 23 samples, regions of interest (ROIs) were then selected based on the fluorescence information and sequentially processed by the microscope automation. Tumor and stromal segmentations were processed for each ROI based on Pan-CK+ and - using image $\mathbf{J}$ scripts as described below. UV LED light was collimated to be reflected from the digital micromirror device (DMD) surface into the microscope objective and focused at the sample tissue with paired segmentation mask (described below). A microcapillary tip connected to a syringe pump primed with buffer solution was moved to each ROI and collected oligos released from the tissue by UV irradiation. These oligos were further quantitated using Nanostring GeoMx ${ }^{\mathrm{TM}}$ Digital Spatial Profiler.

Hybridization of cleaved indexing oligos to NanoString barcoded sequences was performed using the nCounter PlexSet reagents. Oligos were denatured at $95^{\circ} \mathrm{C}$ for 3 to 5 minutes and placed on ice for 2 minutes. A master mix was created by adding $70 \mu \mathrm{L}$ of hybridization buffer and in situ capture probes (ICP) to the PlexSet tube (A-G). A $7 \mu \mathrm{L}$ aliquot of master mix was added to each well in 96-well plate. And denatured protein samples were added to the well and the volume of each well was brought to a final volume of $15 \mu \mathrm{L}$ with DEPC treated water. Hybridizations were performed at $65^{\circ} \mathrm{C}$ overnight in a thermocycler. After hybridization, samples were pooled by column and processed using the nCounter Prep Station and Digital Analyzer as per manufacturer instructions.

Digital counts from tags corresponding to protein probes were analyzed as follows: raw counts were first normalized with internal spike-in controls to account for system variation. These normalized values were then normalized by adjusting for the area of sample illuminated by UV and $\log _{2}$ transformed prior to analysis. Antibodies with mean expression below the mean of the highest IgG control antibody were excluded from analysis.

\section{Sample preparation for ISH Digital Spatial Profiling}

For in situ hybridization, $5 \mu \mathrm{m}$ FFPE sections were freshly cut and mounted onto positive charged slides were baked, deparaffinized, rehydrated in ethanol and washed in PBS using Leica Bond Rxm system. Targets were retrieved for 20 minutes in Leica Epitope Retrieval Solution 2, EDTA PH 9.0 buffer at $100^{\circ} \mathrm{C}$. Tissues were washed with PBS and incubated with $1 \mathrm{ug} / \mathrm{ml}$ proteinase $\mathrm{K}$ in PBS for 15 mins at $37^{\circ} \mathrm{C}$ and washed with PBS. After tissues were removed from the Leica Bond, they were covered with Hybrislip covers (Grace BioLabs) and incubated overnight at $37^{\circ} \mathrm{C}$ with hybridization solutions containing $4 \mathrm{nM}$ of each RNA detection probe; 
$0.1 \mathrm{mg} / \mathrm{mL}$ salmon sperm DNA (Sigma-Aldrich, D7656); 2.5\% dextran sulfate (Sigma-Aldrich, 67578-5G); 0.2\% BSA (ThermoFisher, 37525); 40\% deionized formamide (Ambion, AM9344); 2X SSC (Sigma, S6639). After overnight incubation, HybriSlip covers were gently removed by soaking in $2 \mathrm{X} \mathrm{SSC}+0.1 \%$ Tween 20 and then washed for 5 minutes in $2 \mathrm{X}$ SSC. Two 25-minute stringent washes were performed in $50 \%$ formamide in $2 \mathrm{X} \mathrm{SSC}$ at $37^{\circ} \mathrm{C}$. Tissues were washed twice for 2 minutes each in 2X SSC, and then were blocked with a Nanostring blocking buffer for 30 minutes at room temperature in a humidity chamber. SYTO 13 and fluorescentlyconjugated antibodies targeting Pan-CK and CD3 in blocking buffer were applied for 1 hour at room temperature, then washed three times for 5 minutes each in fresh 2 X SSC. Three of the 25 samples (10167, 10171 and 10230) were omitted from further processing due to poor tissue attachment. Oligos were cleaved and collected using Nanostring GeoMx ${ }^{\mathrm{TM}}$ Digital Spatial Microscope as described above.

\section{Custom UV illumination mask creation}

For each sample, 6 AOIs were selected and the oligonucleotide tags from stroma vs tumor areas were collected and analyzed separately by creating a Pan-CK+ and Pan-CK- UV illumination mask for each AOI. Custom masks were created in ImageJ to define custom regions of interest for UV illumination. These custom masks were used by the DMD device to determine which mirrors would be utilized to direct UV light to tumor and stromal segmentations. For each region of interest, 20x stacked tiff images with four fluorescent channels was split into four separate fluorescent images for custom thresholding. To make tumor segmentation, the image with Pan-CK channel was thresholded manually to match the Pan-CK staining pattern and converted to a binary mask. Remove fluorescent noise by copying a mask generated using "Analyze Particles" with settings size $=0-700$ pixels, circularity $=0.35-1.00$, and show $=$ masks, to Pan-CK binary mask. "Fill holes" was proceeded to fill the nucleus of the tumor cells. "Invert LUT" is to make sure Pan-CK positive region could be UV illuminated. This image was saved as tumor segmentation. Stromal segmentation was generated by inverting tumor segmentation following by being dilated 3 times. Segmentation size is defaulted with $666 \times 666 \mu \mathrm{m}$ box. However, in some ROIs, only center region is designed to be UV radiation exposed. In this case, a centered $665 \mu \mathrm{m}$ diameter circle mask could be added to tumor or stromal segmentations by using "Clear outside" function.

\section{RNA ISH profiling readout by NGS}

PCR was performed on photoreleased oligos collected from each well (corresponding to one AOI) to add Illumina adapter sequences and unique i5 and i7 sample indices. Each PCR reaction used $4 \mathrm{uL}$ of DSP photocleaved oligos, $1 \mathrm{uL}$ of unique-dual indexing PCR primers, $2 \mathrm{uL}$ of Nanostring 5X PCR Master Mix, and 3 uL PCR-grade water. Thermocycling conditions were $37 \mathrm{C}$ for $30 \mathrm{~min}, 50 \mathrm{C}$ for $10 \mathrm{~min}, 95 \mathrm{C}$ for $3 \mathrm{~min}$; 18 cycles of $95 \mathrm{C}$ for $15 \mathrm{sec}, 65 \mathrm{C}$ for $1 \mathrm{~min}, 68 \mathrm{C}$ for $30 \mathrm{sec}$; and 68C $5 \mathrm{~min}$. PCR reactions were pooled and purified twice using AMPure XP beads (Beckman Coulter) according to manufacturer protocol. Pooled libraries were sequenced at $2 \times 38$ base pairs and with the dual index workflow on an Illumina NextSeq to generate 306M raw reads.

For data from the NextSeq instrument, FASTQ files from multiple lanes were merged to generate single files for processing and insure proper removal of PCR duplicates later in the pipeline. Illumina adapter sequences were trimmed using Trim Galore (version 0.4.5) with a minimum base pair overlap stringency of four bases and a base quality threshold of 20. Paired 
end reads were stitched using Paired-End reAd mergeR (PEAR, version 0.9.10) specifying a minimum stitched read length of 24bp and a maximum stitched read length of $28 \mathrm{bp}$. The 14bp UMI sequence was extracted from the stitched FASTQ files from the 5' end of the sequence reads using umi tools (version 0.5.3). The fastq files with extracted UMIs were then aligned to a genome containing the $12 \mathrm{bp}$ reference sequence tags using bowtie2 (version 2.3.4.1) in end-toend mode with a seed length of four. Using a custom python function, the generated SAM files were split in to multiple SAM files based on the tag to which they aligned to limit memory usage when removing PCR duplicates. The split SAM files were converted to bam files, sorted, and index using samtools (version 1.9) with the import, sort, and index options respectively. PCR duplicates were removed from the sorted and indexed bam files using the dedup command from umi tools with an edit distance threshold of three. An edit distance threshold of three was used because using a threshold of one quarter the length of the UMI has been demonstrated to be a conservative threshold. Using custom python functions, the SAM files with PCR duplicates removed were merged for each sample and used to generate digital counts of the tags.

Because each target transcript was counted using multiple probes and tags, outlier counts were removed before generating a consensus count for each target. Outlier tags were identified as those with counts $90 \%$ below the mean of the probe group in at least $20 \%$ of the AOIs analyzed and completely removed them from the analysis. Subsequently we removed tags from the analysis if they were flagged as outliers in at least $20 \%$ of the AOIs analyzed. This was done using the Rosner Test if there were at least 10 probes for the target $(\mathrm{k}=0.2 *$ Number of Probes, alpha $=0.01$ ), or the Grubbs test if there were less than 10 probes for the target. Probes flagged as outliers in less than $20 \%$ of the AOIs analyzed were only removed from the analysis for the AOIs in which they were flagged. Count reported for each target transcript were calculated as the mean of the remaining probes.

The counts for each target transcript were then normalized to the count of the house keeper genes (C1orf43, GPI, OAZ1, POLR2A, PSMB2, RAB7A, SDHA, SNRPD3, TBC1D10B, TPM4, TUBB, UBB). The geometric mean of the house keeper gene counts was calculated for each AOI. These geometric means were then divided by the geometric mean of the geometric mean of the house keepers to generate a normalization factor for each AOI. The counts of the transcripts in each AOI were than multiplied by the associated normalization factor. Expression values were $\log _{2}$ transformed prior to analysis. 


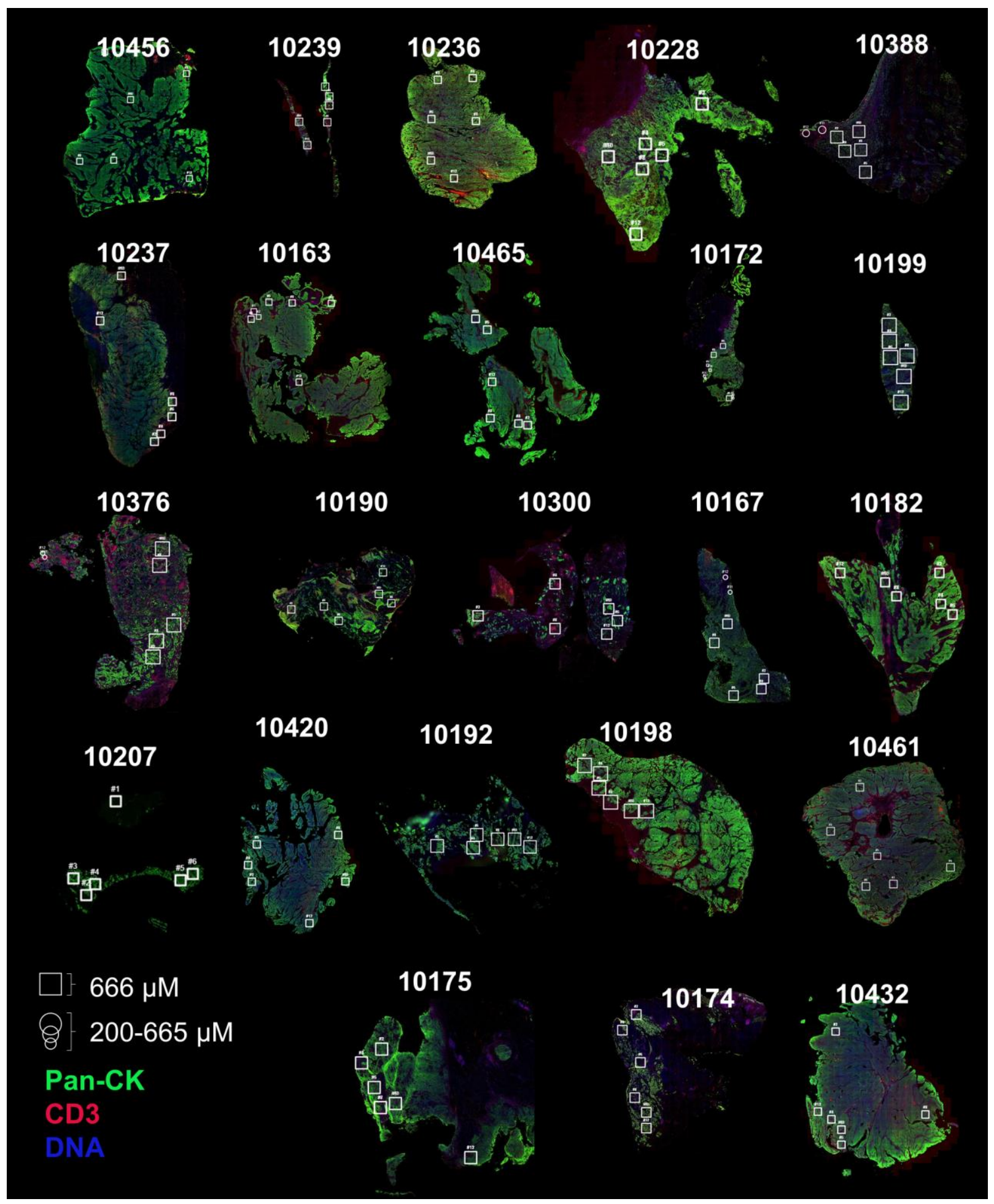

Supplementary Figure 1. ROI placement on guide IF images. Whole-slide IF images are shown for each sample indicating the placement of ROIs throughout the tissue. Each slide is scaled independently, though square ROIs are consistently sized, at $666 \mathrm{uM}$ per ROI. Circular ROIs present in some samples were not used during analysis, as they varied in size and cell type composition. 

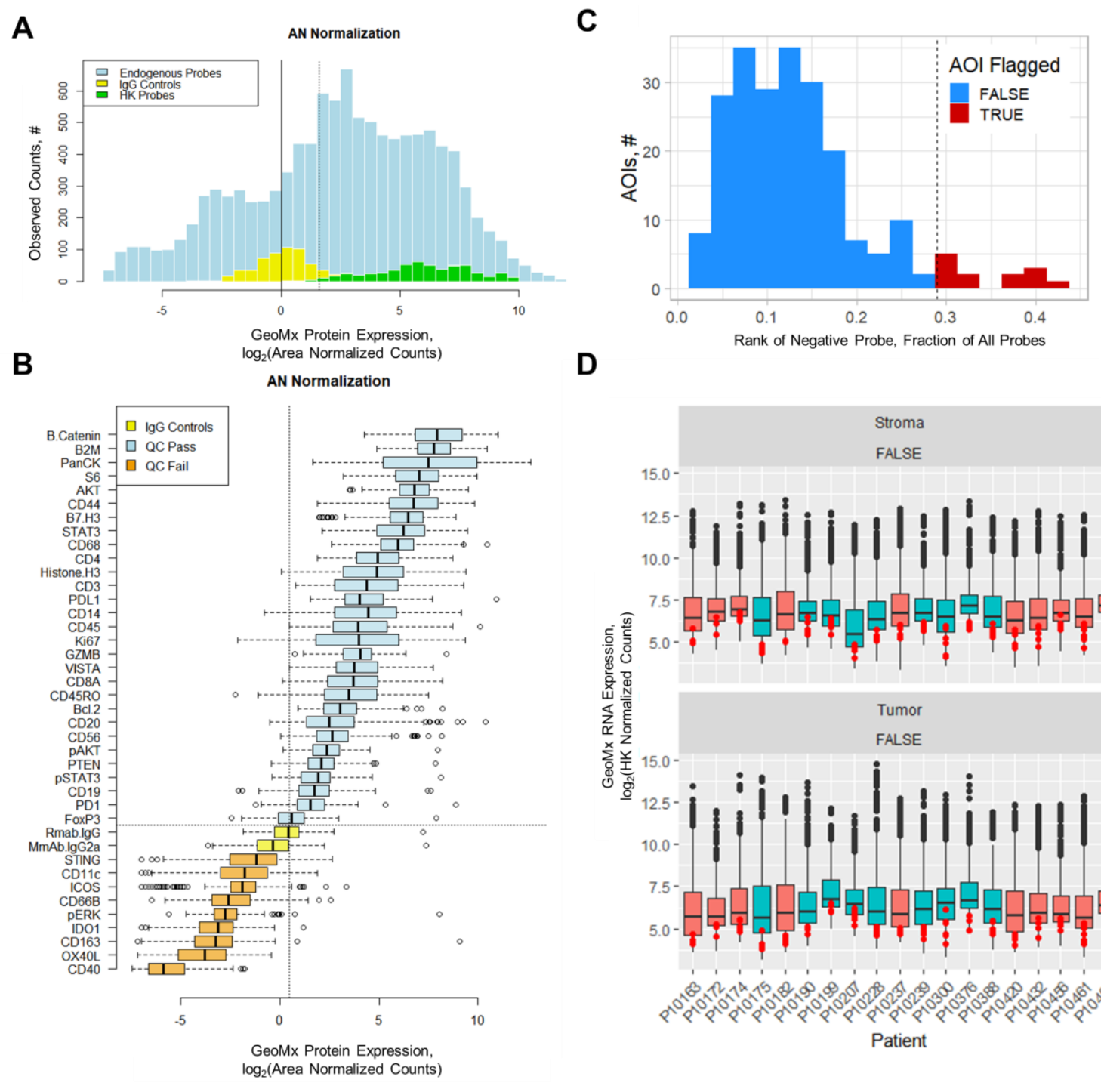

D

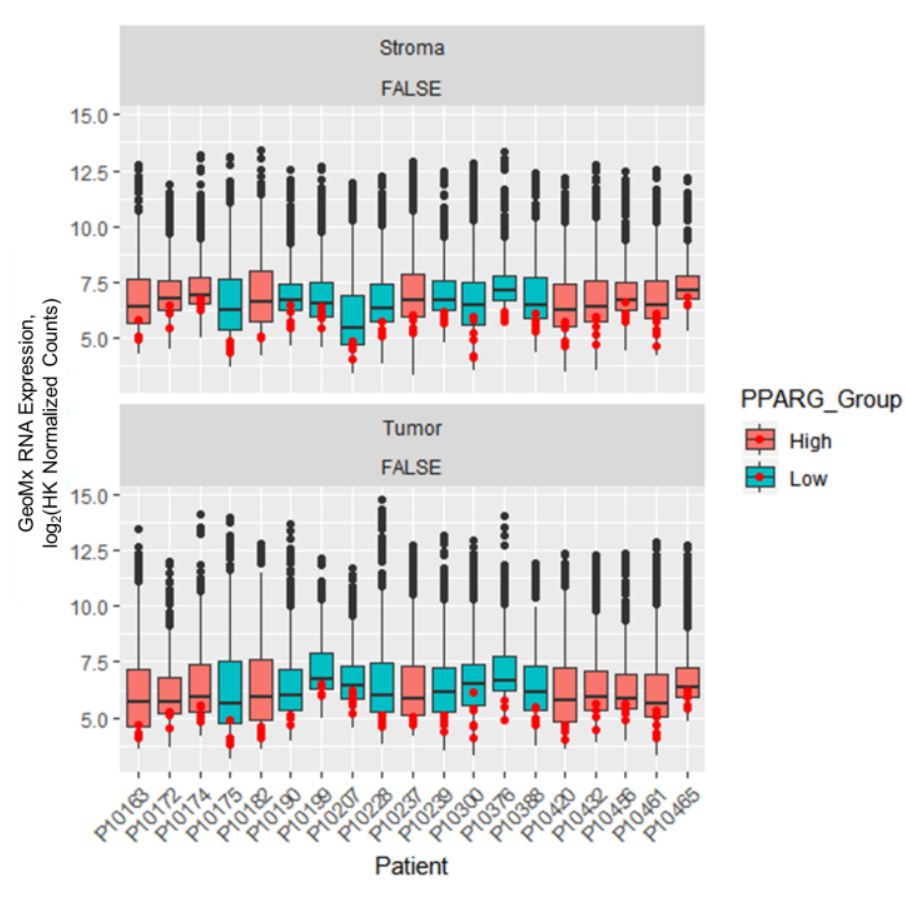

Supplementary Figure 2. QC of GeoMx profiling. (A) Distribution of counts produced across probes from different classes of antibodies. HK probes include Histone H3, and S6. Control probes are Rabbit IgG1 (Rmab.IgG) and Mouse IgG2A (MmAb.IgG2a). All other probes are for endogenous expression detection. (B) Individual probe distribution and QC. Probes falling below the median expression of the Rabbit IgG1 probe were excluded from analysis due to poor signal. (C) Distribution of the rank of the negative control probes from RNA-ISH profiling across AOIs output from RNA-sequencing pipeline. AOIs with high ranking negative probes (e.g. high background samples. $>$ Mean $+2 \sigma)$, were removed from analysis $(n=13)$. (D) Distribution of RNA-ISH expression across all AOIs for all genes measured, aggregated by patient. Red dots indicate the negative control probe expression for each AOI within a patient block. 

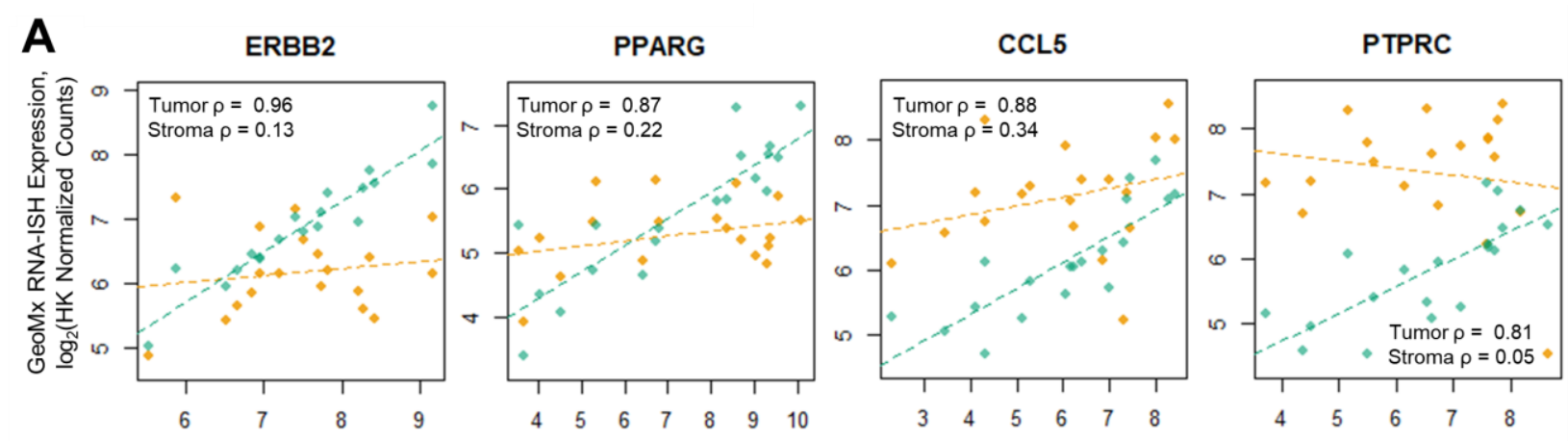

- Tumor AOls Stromal AOIs

Bulk Expression from 10360, $\log _{2}(\mathrm{HK}$ Normalized Counts)
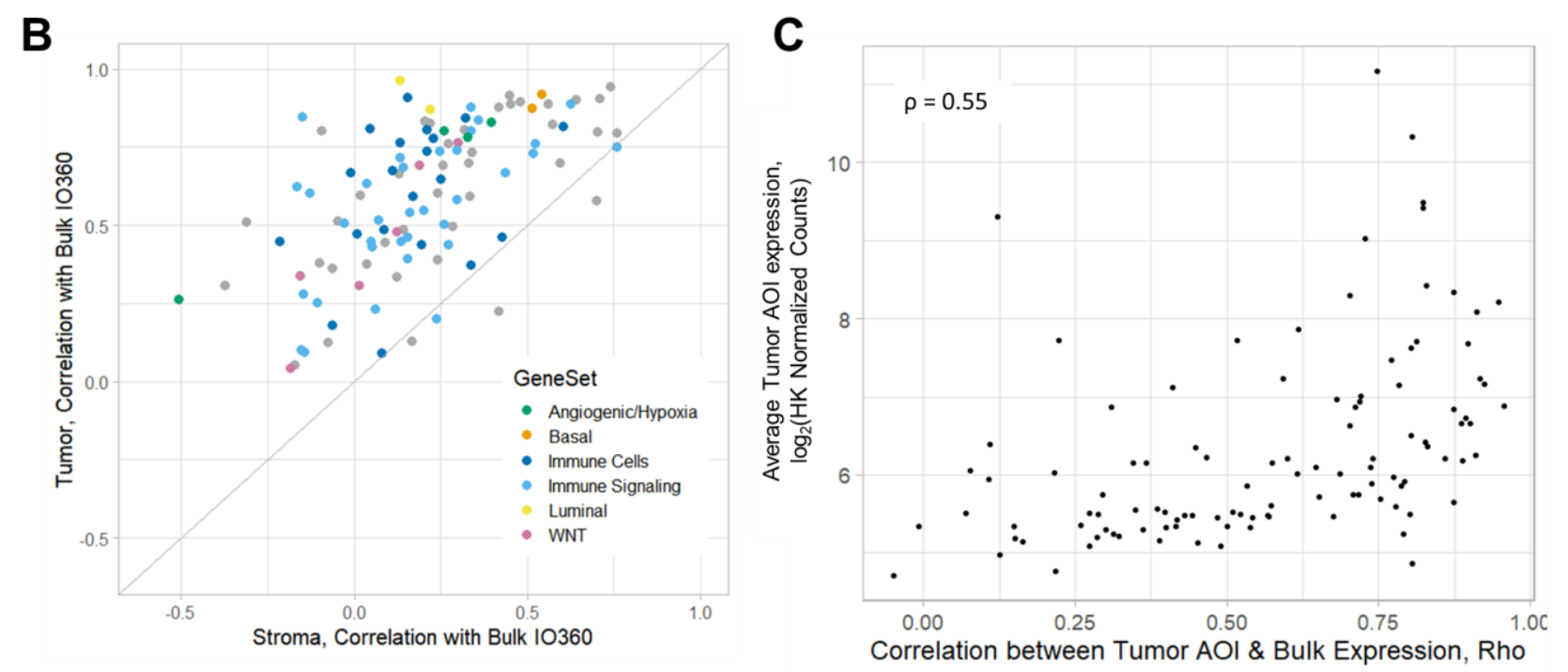

Supplementary Figure 3. Relationship between bulk and GeoMx expression. (A) Example expression patterns for tumor and immune cell related genes. Correlation between average tumor AOI or average stromal AOI expression shown compared to bulk profiling by IO360. (B) Correlation of individual genes with either stroma (x-axis) or tumor (y-axis) expression. Probe colored by gene set membership (C) Expression of tumor AOIs is weakly correlated with how well correlated a given probe is measured in tumor AOIs compared to bulk expression. 


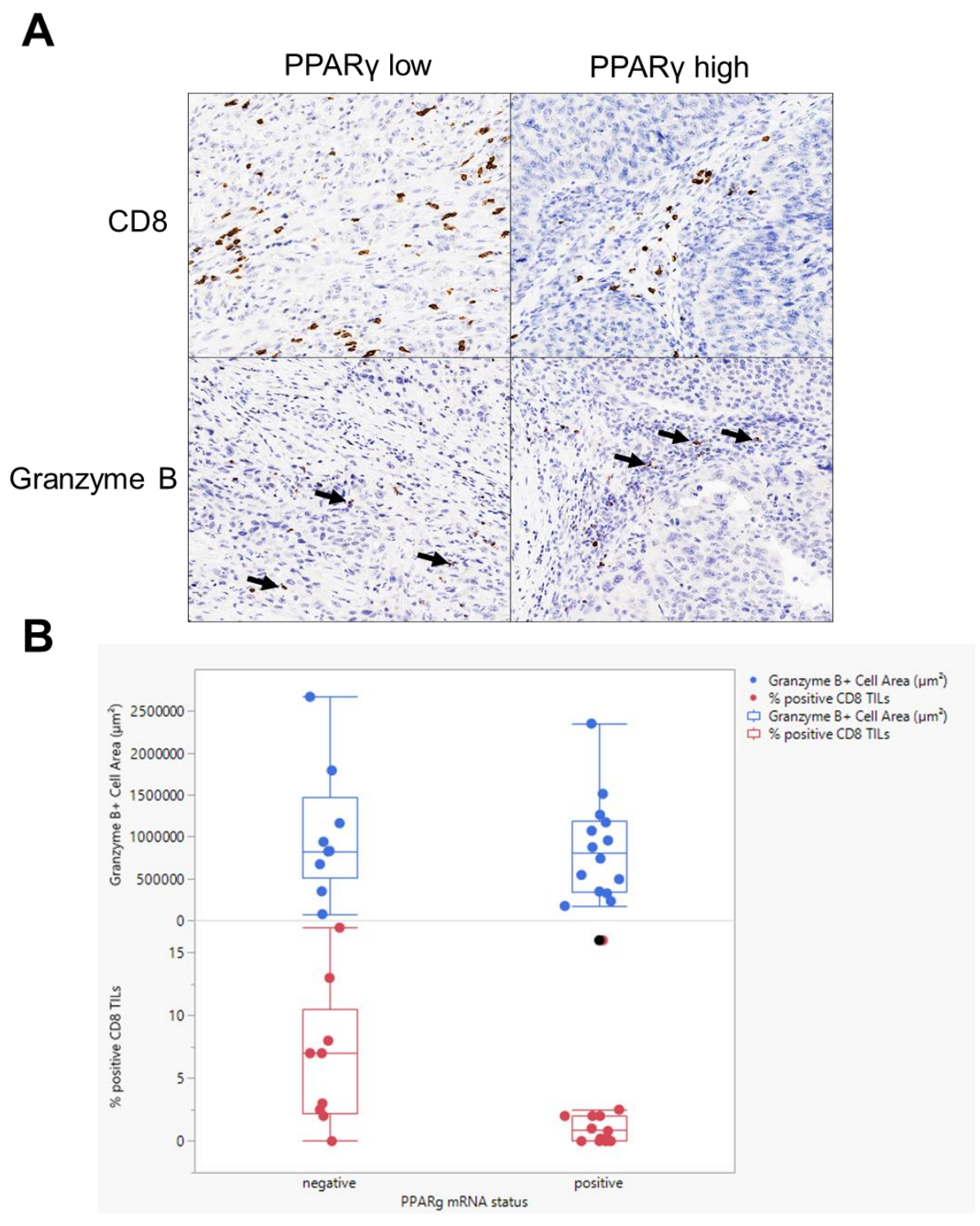

Supplementary Figure 4. IHC Quantification of CD8 and GZMB. (A) Example staining of CD8 and Granzyme B by IHC in a representative PPARg negative and positive tumor. Arrows indicate GZMB+ positive cells. (B) Quantification of samples stained with CD8 and GZMB across the cohort of samples based on PPARG status. 
A

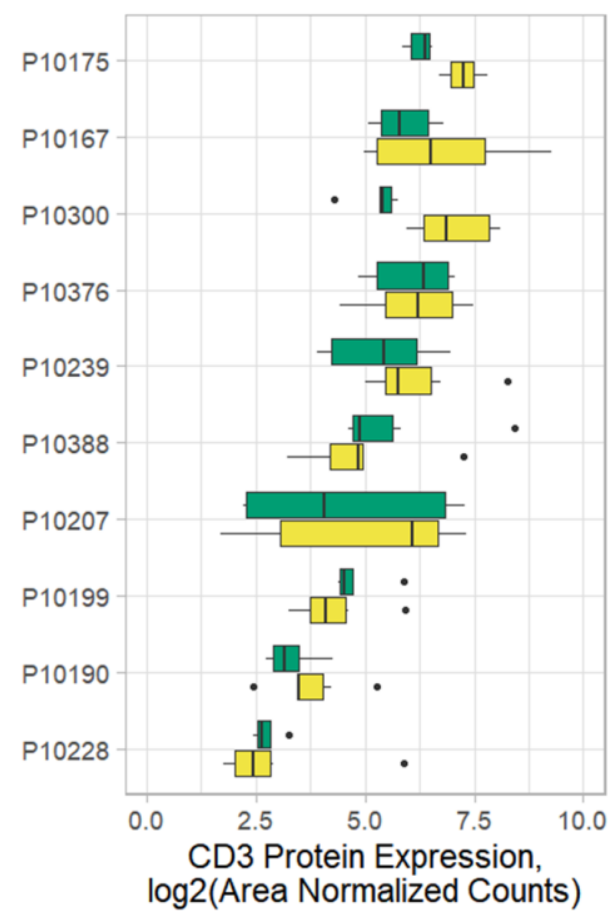

B

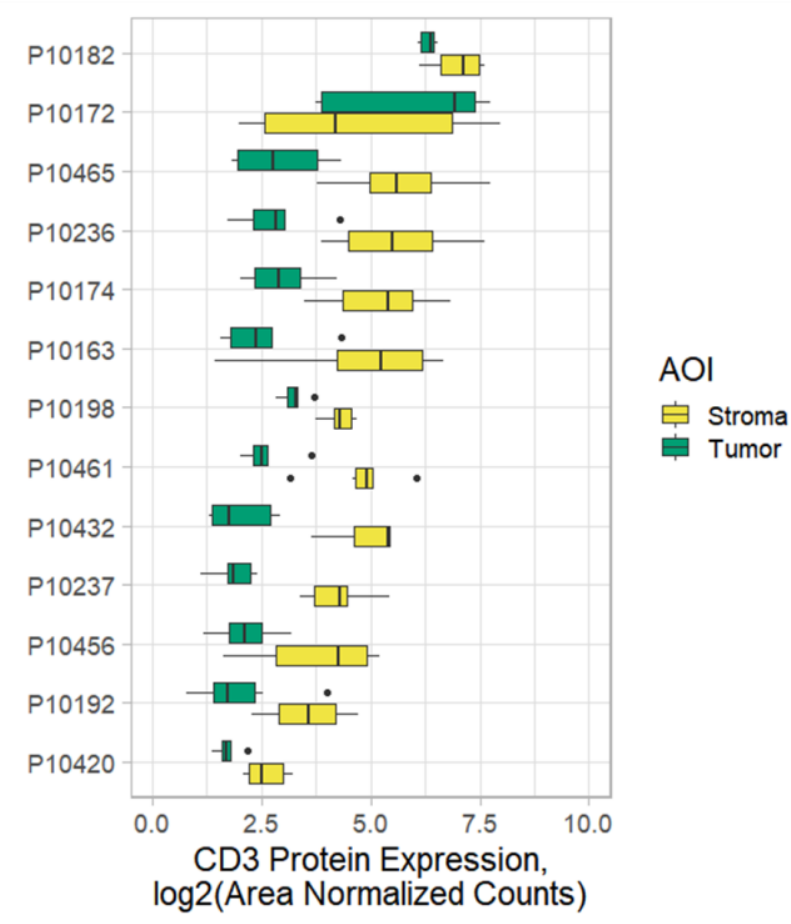

Supplementary Figure 5. AOI expression of CD3 antibody. (A-B) Patient level expression of CD3 for either (A) PPAR $\gamma^{\text {low }}$ or (B) PPAR $\gamma^{\text {high }}$ samples. 